ASC Report No. 39/2009

Scaling in Singular Perturbation Problems: Blowing-up a Relaxation Oscillator

Ilona Gucwa, Peter Szmolyan 


\section{Most recent ASC Reports}

38/2009 Anton Baranov, Harald Woracek

Majorization in de Branges Spaces III. Division of Blaschke Products

37/2009 Philipp Dörsek, Jens Markus Melenk

Adaptive $h p$-FEM for the Contact Problem with Tresca Friction in Linear Elasticity: The Primal-dual Formulation and a Posteriori Error Estimation

36/2009 Philipp Dörsek, Jens Markus Melenk

Adaptive $h p$-FEM for the Contact Problem with Tresca Friction in Linear Elasticity: The Primal Formulation

35/2009 Georg Kitzhofer, Othmar Koch, Gernot Pulverer, Christa Simon, Ewa B. Weinmüller

BVPSUITE, A New MATLAB Solver for Singular Implicit Boundary Value Problems

34/2009 M. Löhndorf, Jens Markus Melenk

Mapping Properties of Helmholtz Boundary Integral Operators and their Application to the hp-BEM

33/2009 Ansgar Jüngel, Stefan Krause, Paola Pietra

Diffusive Semiconductor Moment Equations Using Fermi-Dirac Statistics

32/2009 Ansgar Jüngel

Diffusive and Nondiffusive Population Models

31/2009 Jens Markus Melenk, Stefan Sauter

Wave-Number Explicit Convergence Analysis for Galerkin Discretizations of the Helmholtz Equation (extended version)

30/2009 Matthias Langer, Harald Woracek

A Function Space Model for Canonical Systems with an Inner Singularity

29/2009 Winfried Auzinger, Felix Kramer

On the Stability and Error Structure of BDF Schemes Applied to Linear Parabolic Evolution Equations

Institute for Analysis and Scientific Computing

Vienna University of Technology

Wiedner Hauptstraße 8-10

1040 Wien, Austria

E-Mail: admin@asc.tuwien.ac.at

WWW: http://www.asc.tuwien.ac.at

FAX: $\quad+43-1-58801-10196$

ISBN 978-3-902627-02-5

(C) Alle Rechte vorbehalten. Nachdruck nur mit Genehmigung des Autors.

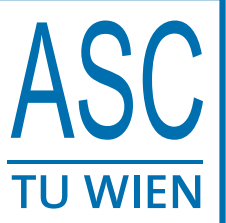




\title{
SCALING IN SINGULAR PERTURBATION PROBLEMS: BLOWING-UP A RELAXATION OSCILLATOR
}

\author{
ILONA GUCWA* AND PETER SZMOLYAN ${ }^{\dagger}$
}

\begin{abstract}
An introduction to some recently developed methods for the analysis of systems of singularly perturbed ordinary differential equations is given in the context of a specific problem describing glycolytic oscillations. In suitably scaled variables the governing equations are a planar system of ordinary differential equations depending singularly on two small parameters $\varepsilon$ and $\delta$. In [20] it was argued that a limit cycle of relaxation type exists for $\varepsilon \ll \delta \ll 1$. The existence of this limit cycle is proven by analyzing the problem in the spirit of geometric singular perturbation theory. The degeneracies of the limiting problem corresponding to $(\varepsilon, \delta)=(0,0)$ are resolved by repeatedly applying the blow-up method. It is shown that the blow-up method leads to a clear geometric picture of this fairly complicated two parameter multi-scale problem.
\end{abstract}

Key words. slow-fast dynamics, relaxation oscillations, geometric singular perturbation theory, blow-up method, slow manifolds

\section{AMS subject classifications.}

1. Introduction. This work is intended to be an introduction to some recently developed methods for the analysis of systems of singularly perturbed ordinary differential equations. Concepts from geometric singular perturbation theory and geometric desingularization based on the blow-up method are explained in the context of a specific problem describing glycolytic oscillations.

The equations we consider are of the form

$$
\begin{aligned}
& x^{\prime}=f(x, y, \varepsilon), \\
& y^{\prime}=\varepsilon g(x, y, \varepsilon)
\end{aligned}
$$

with smooth functions $f$ and $g$ and a small parameter $\varepsilon>0$. The derivative is with respect to the time variable $\tau$. Near points where $f(x, y, \varepsilon)$ is $O(1)$ the variable $x$ varies on the fast scale $\tau$, while near points where $f(x, y, \varepsilon)$ is $O(\varepsilon)$ solutions vary on the slow time scale $t=\tau \varepsilon$. On the time scale $t$ the governing equations are

$$
\begin{aligned}
\varepsilon \dot{x} & =f(x, y, \varepsilon), \\
\dot{y} & =g(x, y, \varepsilon) .
\end{aligned}
$$

Systems of this form arise in many different applications. The most well-known problem of this type is perhaps the Van der Pol oscillator which is the prototype of a slow-fast system exhibiting relaxation oscillations [6], [16]. A detailed description of relaxation oscillations in the context of glycolytic oscillations will be given in Section 2 .

By setting $\varepsilon=0$ in (1.1) we obtain the layer problem

$$
\begin{aligned}
& x^{\prime}=f(x, y, 0), \\
& y^{\prime}=0 .
\end{aligned}
$$

* The research of the first author was supported by the Austrian Science Foundation under grant W8 and the International Max Planck Research School Mathematics in the Sciences in Leipzig.

†The research of the second author was supported by the Austrian Science Foundation under grant W8 and grant Y 42-MAT. 
The corresponding reduced problem on the slow time scale $t$, obtained by setting $\varepsilon=0$ in $(1.2)$, is given by

$$
\begin{aligned}
& 0=f(x, y, 0) \\
& \dot{y}=g(x, y, 0) .
\end{aligned}
$$

The set $S$ defined by the equation $f(x, y, 0)=0$ is called the critical manifold. The critical manifold is the set of equilibria of the layer problem (1.3), whereas the reduced problem (1.4) is a dynamical system on $S$. The critical manifold $S$ is not always a manifold in the strict sense, since points of self-intersection or other singularities may arise. In a neighborhood of any point in $S$ where the Jacobian $f_{x}(x, y, 0)$ is nonsingular the equation $f(x, y, 0)=0$ can be solved for $x=h(y)$ by the implicit function theorem. In this situation the reduced problem (1.4) is described by the equation

$$
\dot{y}=g(h(y), y, 0)
$$

In many problems valuable information on the dynamics of system (1.1) with $\varepsilon$ small can be obtained by analyzing and suitably combining the dynamics of the layer problem and the reduced problem. It is natural to expect that the layer problem is an approximation of the fast dynamics and that the reduced problem is an approximation of the slow dynamics. In many situations higher order approximations are obtained by the method of matched asymptotic expansions and (less often) the validity of the expansions is proven [6], [11], [16], [17].

During the last twenty years another more qualitative approach based on methods from dynamical systems theory known as geometric singular perturbation theory has been developed. This approach goes back to Fenichel [4], as an introduction we recommend the survey [10], where also references to numerous applications can be found. However, Fenichel theory applies only to normally hyperbolic parts of the critical manifold $S$, where the Jacobian $f_{x}(x, y, 0)$ is uniformly hyperbolic, i.e. its spectrum is uniformly bounded away from the imaginary axis.

Points on the critical manifold $S$ where the Jacobian $f_{x}(x, y, 0)$ is non-hyperbolic, i.e. $f_{x}(x, y, 0)$ has a zero eigenvalue or a purely imaginary eigenvalue, are a major source of difficulties in all approaches. A zero eigenvalue of $f_{x}(x, y, 0)$ at a point in $S$ is typically related to a singularity of the critical manifold $S$. The most common singularity in that context are fold points corresponding to a saddle-node bifurcation of $S$. In the method of matched asymptotic expansions these singularities of $S$ lead to complicated asymptotic expansions containing fractional powers and logarithms of $\varepsilon$, see e.g.[6], [16], and also [5], [13].

For a long time it was unclear how to extend geometric singular perturbation theory to fold points and other non-hyperbolic points of the critical manifold $S$. After the pioneering work of Dumortier and Roussarie [1] it turned out that the blow-up method is a powerful tool in the analysis of singular perturbation problems with nonhyperbolic points. We refer to [13] for a detailed introduction to the blow-up method in the context of singularly perturbed planar folds. In this approach a fold point is considered as a very degenerate equilibrium of the extended system in $\mathbb{R}^{3}$ obtained by adding the equation $\varepsilon^{\prime}=0$ to a planar system of the form (1.1). This degenerate equilibrium is blown-up to a sphere by introducing suitably weighted spherical coordinates. Since an equilibrium is blown-up, the blown-up vector field vanishes on the sphere. After dividing out a suitable power of the radial variable, a sufficiently 
non-degenerate flow on the sphere is obtained to allow a complete analysis. A brief introduction to geometric singular perturbation theory and the blow-up method in the context of planar systems with folded critical manifolds is given in Appendix $A$. For more general background on dynamical systems, we refer to [7].

By now this method has found numerous applications in the analysis of the dynamics associated with non-hyperbolic points of singularly perturbed differential equations, see e.g. [1], [2], [3], [12], [14], [15], [18], [21], [22]. In many of these applications degenerate points are blown-up to spheres, however there are also works where higher dimensional degenerate objects are blown-up, e.g. non-hyperbolic curves are blownup to cylinders [8], [9], [19]. In some of these works the blow-up method has to be used iteratively, i.e. several consecutive blow-ups have to be used to obtain a complete desingularization. More explicitly this means that if a certain blow-up leads to a less degenerate problem which however still has degenerate points, these points can be treated by additional blow-ups [8].

In this paper we explain some of the above mentioned features of the geometric approach to singular perturbation problems and especially of the blow-up method in the context of the specific example (1.6). In addition to the intrinsic interest in system (1.6) as a model for glycolytic oscillations there are several other motivations for choosing this example. We believe that the problem under consideration is wellsuited to serve as an introduction to the blow-up method in the context of a non-trivial application. In particular, the iterative nature of the procedure is clearly visible. Furthermore, the blow-up approach to this two parameter problem could be useful in other problems depending singularly on several parameters. It will turn out that the parameter $\varepsilon$ mainly affects the slow-fast structure while the parameter $\delta$ mainly influences the geometry and the singularities of the critical manifold. We will show that the blow-up method leads to a clear geometric picture of this fairly complicated two parameter multi-scale problem. Last, but not least, all necessary computations can be easily carried out explicitly. We tried to give a careful explanation of the blow-up procedure with respect to $\delta$, i.e. we give all the details leading to Theorem 4.5 and the geometry shown in Figure 4.6. The subsequent perturbation analysis with respect to $\varepsilon$ is also carried out, but requires a certain background in dynamical systems theory.

We now turn to the description of the specific problem which will be studied in this work. In [20] Segel and Goldbeter analyzed a model describing glycolytic oscillations. In dimensionless variables the governing equations have the form

$$
\begin{aligned}
\rho \alpha^{\prime} & =\mu-\phi(\alpha, \gamma) \\
\gamma^{\prime} & =\lambda \phi(\alpha, \gamma)-\gamma
\end{aligned}
$$

where $\alpha$ and $\gamma$ denote certain substrate and product concentrations and

$$
\phi(\alpha, \gamma)=\frac{\alpha^{2}(\gamma+1)^{2}}{L+\alpha^{2}(\gamma+1)^{2}}
$$

The equations contain four positive parameters where $L$ and $\lambda$ turn out to be large and satisfy $\lambda \ll L$, while $\mu$ and $\rho$ are of moderate size.

In their analysis of system (1.6) Segel and Goldbeter applied what they call the method of scaling. In particular they identified a small parameter

$$
\varepsilon:=\sqrt{\frac{\lambda}{L}}
$$


which causes the slow-fast structure of system (1.6). It turns out that system (1.6) exhibits classical relaxation oscillations in the limit of large $L$ and fixed $\lambda$. However, the main interest in [20] lies in the situation where $L$ and $\lambda$ are both large. In this case, the asymptotics of system (1.6) becomes more complicated. By considering several different scaling regimes Segel and Goldbeter argued that the condition

$$
\sqrt{\frac{\lambda}{L}} \ll \frac{1}{\sqrt{\lambda}} \ll 1
$$

implies the existence of a relaxation cycle.

In this work we complement the reasoning given in [20] by proving that condition (1.8) indeed implies the existence of a relaxation cycle of system (1.6). We will rewrite system (1.6) in the standard form (1.1) of singularly perturbed problems, which we then examine geometrically in the spirit described above. By a suitable scaling of the variables $\alpha$ and $\gamma$ we rewrite system (1.6) in the form

$$
\begin{aligned}
a^{\prime} & =\varepsilon\left(a^{2} b^{2}(\mu-1)+\mu \delta^{2}\right), \\
b^{\prime} & =a^{2} b^{2}(1-b)+\delta^{2}\left(a^{2} b^{2}-b+\delta^{2}\right),
\end{aligned}
$$

where $(a, b)$ correspond to $(\alpha, \gamma), \varepsilon$ is given by equation (1.7) and

$$
\delta:=\lambda^{-1 / 2} \text {. }
$$

Hence, $a$ is the slow variable and $b$ is the fast variable with respect to $\varepsilon$. In our notation condition (1.8) has the from

$$
\varepsilon \ll \delta \ll 1
$$

We now briefly outline our approach to the analysis of system (1.6). Setting $\varepsilon=0$ and $\delta=0$ in system (1.9) gives the layer problem

$$
\begin{aligned}
& a^{\prime}=0, \\
& b^{\prime}=a^{2} b^{2}(1-b) .
\end{aligned}
$$

The corresponding critical manifold $S^{0}$ defined by $a^{2} b^{2}(1-b)=0$ consists of the lines $a=0, b=0$, and $b=1$, which we denote by $l_{b}, l_{a}$, and $l_{h}$, respectively. Since the zeros $a=0$ and $b=0$ have multiplicity two, the lines $l_{a}$ and $l_{b}$ are non-hyperbolic lines of equilibria, whereas the line $l_{h}$ corresponding to the simple zero $b=1$ is normally hyperbolic. Hence, this limiting problem is quite degenerate and the structure of the relaxation oscillations is not visible at all.

The main point of this work is to show that the blow-up method is well suited to overcome these difficulties. It turns out that two blow-ups of the degenerate critical manifold $S^{0}$ (with respect to $\delta$ ) lead to a complete desingularization of the problem such that uniform results in $\varepsilon$ become possible. In this approach the degenerate lines $a=0$ and $b=0$ are blown-up to cylinders by rewriting the original $(a, b, \delta)$ variables in suitable cylindrical variables. In the blown-up geometry the existence of the relaxation cycle can be proven. Not surprisingly the scaling regimes of Segel and Goldbeter are also recovered in the course of the analysis.

The article is organized as follows. In Section 2 we discuss basic properties of the model, in particular a preliminary slow-fast analysis is given. A more detailed analysis of three scaling regimes is performed in Section 3. In Section 4 the blowup analysis is carried out and the existence of a periodic orbit of relaxation type is 
proven. To make the paper self-contained a brief introduction to geometric singular perturbation theory in the context of planar systems with folded critical manifolds is given in Appendix $A$.

\section{The model and its singular limits.}

2.1. Basic properties and scaling. We begin by reproducing a numerical simulation of system (1.6) from [20] for the parameter values $L=5 \times 10^{6}, \rho=2.5$, $\lambda=40, \mu=0.15$. The results shown in Figure 2.1 indicate the existence of an attracting periodic solution of relaxation type.

Figure 2.1 illustrates the nullclines in the $(\alpha, \gamma)$-plane. Straightforward calculations in [20] prove the following properties. The condition $\lambda>8$ guarantees that the $\gamma$ nullcline is a folded curve with fold points $B$ and $D$. For $\mu<1$ the nullclines intersect just once in the unique equilibrium point of the system. The steady state is unstable when it lies between the fold points $B$ and $D$ of the $\gamma$-nullcline. This is the situation where a limit cycle is expected to exist. For $\mu=0.15$ we have this situation. The numerically computed attracting limit cycle is shown in Figure 2.1. The limit cycle is of relaxation type, i.e. the solution follows the left branch of the $\gamma$-nullcline until it reaches the fold point $B$, from there the solution jumps to a point $C$ on the right branch of the $\gamma$-nullcline, follows the right branch until it reaches the fold point $D$, from where it jumps back to a point $A$ on the left branch. Here we have followed the notation of [20], where more details can be found. Following [20] our analysis will

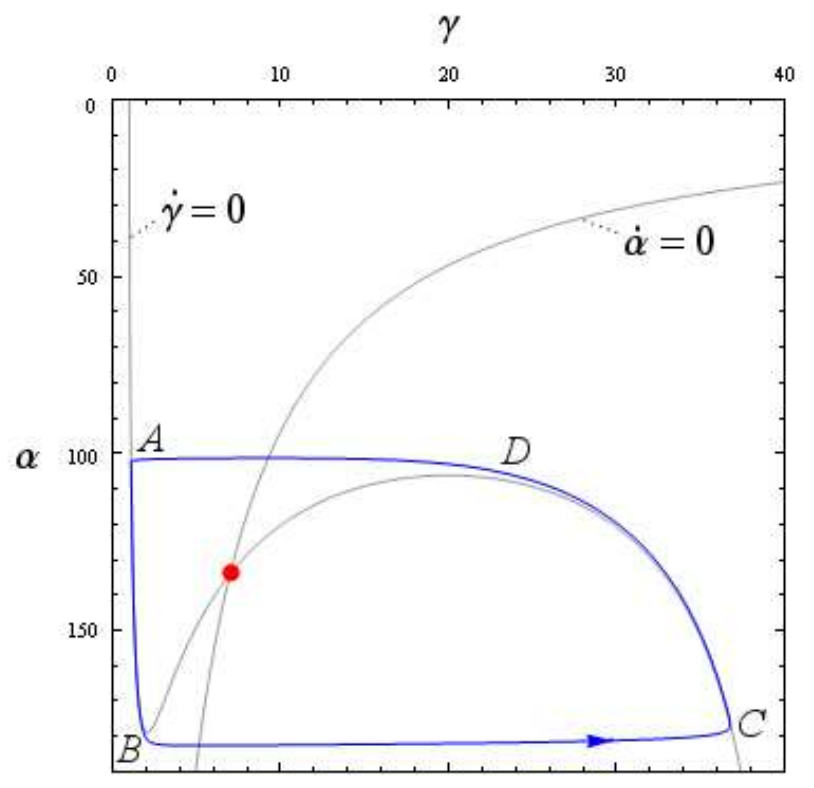

FiguRE 2.1. Nullclines and numerically computed limit cycle for $\mu=0.15, L=5 \times 10^{6}, \lambda=40$.

be based on the assumption that $L$ and $\lambda$ are large, where $L$ is larger then $\lambda$ in the sense of condition (1.8). The scaling analysis in [20] is based on the following scaling properties of the points $A, B, C, D$ with respect to $L$ and $\lambda$. To leading order these 
points are

$$
A \approx\left(\frac{2 \sqrt{L}}{\lambda}, 1\right), \quad B \approx\left(\frac{1}{2} \sqrt{\frac{L}{\lambda}}, 1\right), \quad C \approx\left(\frac{1}{2} \sqrt{\frac{L}{\lambda}}, \lambda\right), \quad D \approx\left(\frac{2 \sqrt{L}}{\lambda}, \frac{\lambda}{2}\right) .
$$

To simplify notation we make the shift $\alpha=\hat{a}, \gamma=\hat{b}-1$, which does not affect the validity of (2.1). Based on the orders of magnitude of $B$ and $C$ we introduce the scaling

$$
\hat{a}=\sqrt{\frac{L}{\lambda}} a, \quad \hat{b}=\lambda b .
$$

For the rest of the paper we set $\rho=1$ and restrict attention to the physically meaningful range of the variables $a, b \geq 0$. In these variables system (1.6) has the form

$$
\begin{aligned}
a^{\prime} & =\varepsilon\left[\mu-\frac{a^{2} b^{2}}{\delta^{2}+a^{2} b^{2}}\right], \\
b^{\prime} & =\frac{a^{2} b^{2}}{\delta^{2}+a^{2} b^{2}}-b+\delta^{2},
\end{aligned}
$$

where $\varepsilon$ and $\delta$ are defined by equations (1.7) and (1.10), respectively. For computational purposes we prefer to write system (2.3) in the equivalent polynomial form

$$
\begin{aligned}
a^{\prime} & =\varepsilon\left[a^{2} b^{2}(\mu-1)+\mu \delta^{2}\right], \\
b^{\prime} & =a^{2} b^{2}(1-b)+\delta^{2}\left(a^{2} b^{2}-b+\delta^{2}\right) .
\end{aligned}
$$

System (2.4) is obtained by multiplying the right hand side of (2.3) by the nonvanishing factor $\delta^{2}+a^{2} b^{2}$, which leaves the orbits of the system unchanged. The resulting rescaled time variable is denoted by $\tau$.

System (2.4) with $\varepsilon$ small is in the standard form of slow-fast systems with slow variable $a$ and fast variable $b$. By transforming to the slow time scale $t=\varepsilon \tau$ the equivalent system

$$
\begin{aligned}
\dot{a} & =a^{2} b^{2}(\mu-1)+\mu \delta^{2}, \\
\varepsilon \dot{b} & =a^{2} b^{2}(1-b)+\delta^{2}\left(a^{2} b^{2}-b+\delta^{2}\right)
\end{aligned}
$$

is obtained, where the derivative is with respect the slow time scale $t$.

2.2. Slow-fast analysis of classical relaxation oscillations for fixed $\delta>0$. Setting $\varepsilon=0$ in (2.4) and (2.5) defines two limiting systems: the layer problem

$$
\begin{aligned}
& a^{\prime}=0, \\
& b^{\prime}=a^{2} b^{2}(1-b)+\delta^{2}\left(a^{2} b^{2}-b+\delta^{2}\right),
\end{aligned}
$$

and the reduced problem

$$
\begin{aligned}
\dot{a} & =a^{2} b^{2}(\mu-1)+\mu \delta^{2}, \\
0 & =a^{2} b^{2}(1-b)+\delta^{2}\left(a^{2} b^{2}-b+\delta^{2}\right) .
\end{aligned}
$$

The equation

$$
a^{2} b^{2}(1-b)+\delta^{2}\left(a^{2} b^{2}-b+\delta^{2}\right)=0
$$

defines the critical manifold $S$, which is of crucial importance for problems of this type, because it controls the slow and the fast dynamics as explained in the introduction. 
Since $S$ is precisely the $\gamma$-nullcline in the new variables, it is again a folded curve for $\delta<1 / \sqrt{8}$. The fold points of $S$ are still denoted by $B$ and $D$, respectively, see Figure 2.2. The points $B$ and $D$ divide $S$ into an attracting left branch $S_{l}$, a repelling middle branch $S_{m}$, and an attracting right branch $S_{r}$, where attracting and repelling refer to the stability properties of points in $S$ considered as steady states of the layer problem (2.6). Since the $a$-nullcline intersects $S$ only once in the middle branch $S_{m}$ with $\dot{a}>0$ on the left of this nullcline, the variable $a$ increases for the reduced flow on $S_{l}$ and decreases on $S_{r}$. Hence, we obtain the following standard scenario of relaxation oscillations for fixed $\delta>0$. A solution starting close to the point $A$ is attracted by $S_{l}$, follows the reduced dynamics on $S_{l}$ until it reaches the point $B$, jumps from the point $B$ to the point $C \in S_{r}$, follows the reduced dynamics on $S_{r}$ until it reaches the point $D$ from where it finally jumps back to the point $A$. The closed curve consisting of the segment of $S_{l}$ from $A$ to $B$, the heteroclinic orbit of the layer problem (2.6) connecting $B$ to $C$, the segment of $S_{r}$ from $C$ to $D$, and the heteroclinic orbit of the layer problem (2.6) connecting $D$ to $A$ is called a singular cycle $\Gamma_{0}$.

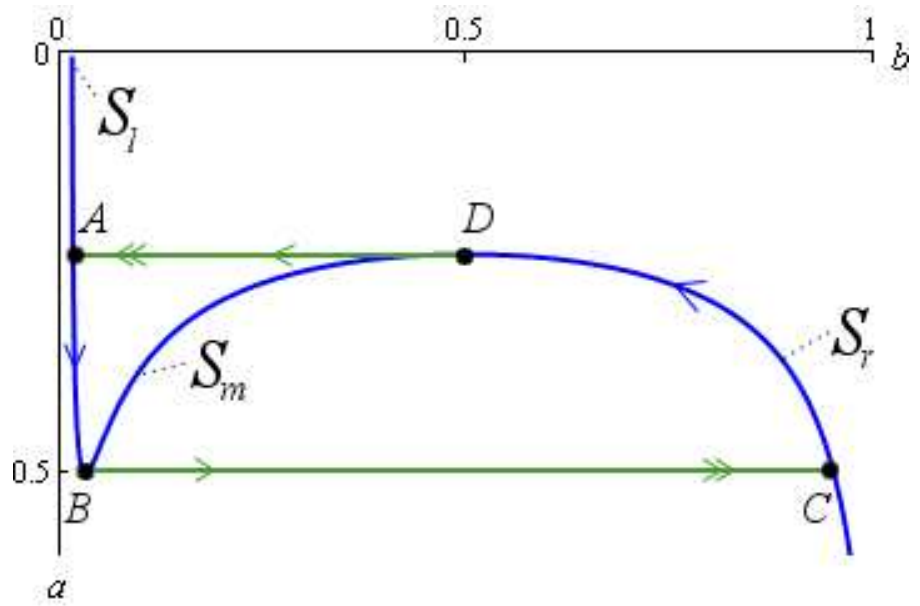

Figure 2.2. Critical manifold $S$ and singular cycle $\Gamma_{0}$.

REMARK 2.1. In the figures of this paper the dynamics of the certain reduced problems and corresponding layer problems are shown simultaneously. Hyperbolic behavior of the layer problem is indicated by double arrows, e.g. near points $A$ and $C$, while a single arrow indicates non-hyperbolic behavior of the layer problem, e.g. near points $B$ and D, see e.g. Figure 2.2.

The situation is essentially as in the classical Van der Pol oscillator [6], [16]. In these works and the references therein problems of this type have been analyzed by the method of matched asymptotic expansions. The main difficulty in the analysis of relaxation oscillations is the analysis of the behavior of the solutions near the fold points $B$ and $D$. During the last decade it became clear how to approach these problems in the framework of geometric singular perturbation theory by combining standard Fenichel theory [4] with the blow-up method [1], [13]. The relevant results from geometric singular perturbation theory and the treatment of fold points by the blow-up method are summarized in Appendix $A$. Theorem 2.1 in [14] implies the existence of an attracting relaxation cycle of system (2.5) for fixed $\delta>0$ and sufficiently small $\varepsilon$. 
Our main interest will be the analysis of a certain limit where $\varepsilon$ and $\delta$ tend to zero simultaneously. For later reference we now change the notation for all the objects introduced in this section by explicitly adding the parameter $\delta$, i.e. the critical manifold is denoted $S^{\delta}$, the points defining the singular cycle $\Gamma_{0}^{\delta}$ are denoted by $A^{\delta}$, $B^{\delta}, C^{\delta}$ and $D^{\delta}$, where $\delta \in(0,1 / \sqrt{8})$. The above analysis for $\varepsilon \rightarrow 0$ for $\delta$ fixed is highly non-uniform with respect to $\delta$. In particular, the geometry of the critical manifold $S^{\delta}$ depends singularly on $\delta$ for $\delta \rightarrow 0$.

2.3. The case $\varepsilon, \delta$ small. It turns out that the limit $(\varepsilon, \delta) \rightarrow(0,0)$ for system (2.4) is more singular then the limit $\varepsilon \rightarrow 0$ in Subsection 2.2. In [20] it is argued that condition (1.8) is sufficient for the existence of a relaxation cycle. We will show that this condition indeed implies the existence of a relaxation cycle.

For $(\varepsilon, \delta)=(0,0)$ system $(2.4)$ has the simple form

$$
\begin{aligned}
& a^{\prime}=0, \\
& b^{\prime}=a^{2} b^{2}(1-b),
\end{aligned}
$$

which is the layer problem (2.6) with $\delta=0$. This limiting problem is dynamically fairly degenerate as shown in the following. System (2.8) has a set of equilibria defined by the equation $a^{2} b^{2}(1-b)=0$ which we denote by $S^{0}$. The critical manifold $S^{0}$ of (2.8) consists of the lines $a=0, b=0$, and $b=1$, which we denote by $l_{b}, l_{a}$, and $l_{h}$, respectively. Since the zeros $a=0$ and $b=0$ have multiplicity two, the lines $l_{a}$ and $l_{b}$ are non-hyperbolic lines of equilibria, whereas the line $l_{h}$ corresponding to the simple zero $b=1$ is normally hyperbolic. The family of critical manifolds $S^{\delta}$ from Subsection 2.2 converges to (the more degenerate) critical manifold $S^{0}$ as $\delta \rightarrow 0$ in a singular way.

The lines of equilibria $l_{a}$ and $l_{h}$ are connected by heteroclinic orbits, i.e. an equilibrium $\left(a_{0}, 0\right) \in l_{a}$ is connected to the equilibrium $\left(a_{0}, 1\right) \in l_{h}$ by an orbit of the layer problem lying on the straight line $a=a_{0}$, see Figure 2.3. This very degenerate situation allows to define many singular cycles, consisting of segments of $l_{h}, l_{b}, l_{a}$, and one heteroclinic orbit of the layer problem. Fenichel theory applies near the normally hyperbolic line $l_{h}$, but we have no control of the behavior of the non-hyperbolic lines $l_{a}$ and $l_{b}$ for $(\varepsilon, \delta) \neq(0,0)$.

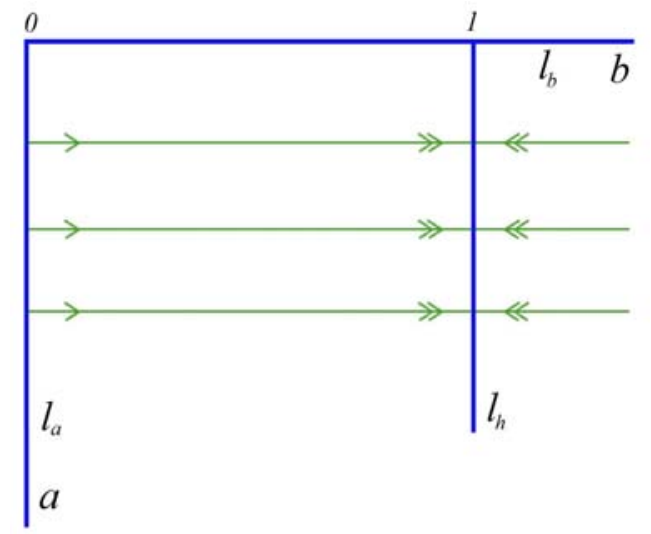

Figure 2.3. Critical manifold $S^{0}$ of system (2.6).

REMARK 2.2. In view of the scaling properties (2.1) of the points $A, B, C, D$ the collapse of the folded critical manifold $S^{\delta}$ of system (2.4) for $\delta>0$ to the more 
singular "manifold" $S^{0}=l_{a} \cup l_{b} \cup l_{h}$ for $\delta=0$ is not surprising, i.e. roughly speaking $S_{l}^{\delta}$ and the left half of $S_{m}^{\delta}$ are compressed onto $l_{a}$, the right half of $S_{m}^{\delta}$ and the lower part of $S_{r}^{\delta}$ are compressed onto $l_{b}$, while the upper part of $S_{r}^{\delta}$ converges to the line $l_{h}$.

3. Scaling regimes. Essential parts of the sought limit cycle are "hidden" in the non-hyperbolic lines $l_{a}$ and $l_{b}$. To make these parts visible we use appropriate re-scalings corresponding to Regimes 1-3 below. The starting point for the scaling analysis are the equations (2.4), where $\varepsilon$ plays the role of a singular perturbation parameter causing the slow-fast structure, while $\delta$ affects mainly the shape of the critical manifold $S^{\delta}$ corresponding to $\varepsilon=0$. It turns out that in Regime 3 the slowfast structure persists only if the assumption $\varepsilon \ll \delta \ll 1$ is used. To make this assumption more explicit we will write

$$
\varepsilon=\delta \tilde{\varepsilon}
$$

in certain places, where $\tilde{\varepsilon} \geq 0$ is still considered as a small parameter.

3.1. Regime 1, $a=O(1), b=O\left(\delta^{2}\right)$. We introduce the scaling

$$
a=a_{1}, \quad b=\delta^{2} b_{1} .
$$

In these variables system (2.4) has the form

$$
\begin{aligned}
a_{1}^{\prime} & =\varepsilon \delta^{2}\left[\delta^{2} a_{1}^{2} b_{1}^{2}(\mu-1)+\mu\right] \\
\delta^{2} b_{1}^{\prime} & =\delta^{4} a_{1}^{2} b_{1}^{2}\left(1-\delta^{2} b_{1}\right)+\delta^{2}\left(\delta^{4} a_{1}^{2} b_{1}^{2}-\delta^{2} b_{1}+\delta^{2}\right) .
\end{aligned}
$$

By rescaling time we cancel a factor of $\delta^{2}$ on the right hand side to obtain the equivalent system

$$
\begin{aligned}
& a_{1}^{\prime}=\varepsilon\left[\delta^{2} a_{1}^{2} b_{1}^{2}(\mu-1)+\mu\right], \\
& b_{1}^{\prime}=a_{1}^{2} b_{1}^{2}\left(1-\delta^{2} b_{1}\right)+\delta^{2} a_{1}^{2} b_{1}^{2}-b_{1}+1 .
\end{aligned}
$$

This system is a slow-fast system for $\varepsilon$ small which depends regularly on $\delta \in\left[0, \delta_{0}\right]$ for $\left(a_{1}, b_{1}\right)$ bounded.

For $\varepsilon=0$ we obtain a new layer problem depending on $\delta$

$$
\begin{aligned}
a_{1}^{\prime} & =0 \\
b_{1}^{\prime} & =a_{1}^{2} b_{1}^{2}\left(1-\delta^{2} b_{1}\right)+\delta^{2} a_{1}^{2} b_{1}^{2}-b_{1}+1 .
\end{aligned}
$$

By setting $\delta=0$ problem (3.5) simplifies to

$$
\begin{aligned}
& a_{1}^{\prime}=0 \\
& b_{1}^{\prime}=a_{1}^{2} b_{1}^{2}-b_{1}+1 .
\end{aligned}
$$

The critical manifold $S_{1}^{0}$ of this system defined by $a_{1}^{2} b_{1}^{2}-b_{1}+1=0$ has an attracting branch $S_{l, 1}^{0}$ corresponding to $b_{1}<2$, and a repelling part $S_{m, 1}^{0}$ corresponding to $b_{1}>2$ which are separated by a fold point $B_{1}^{0}=(1 / 2,2)$. The branch $S_{m, 1}^{0}$ is asymptotic to the $b_{1}$-axis as $b_{1} \rightarrow \infty$. An orbit which starts close to the $b_{1}$-axis is rapidly attracted by $S_{l, 1}^{0}$, follows the reduced dynamics until it reaches the fold point from where it jumps to the right along the orbit $\omega$ of the layer problem, see Figure 3.1. Thus, we have the situation of a jump point as described in Appendix $A$. We specify Regime 1 by the conditions $a_{1} \in[-0.1,0.6]$ and $b_{1} \in\left[0, \beta_{1}\right]$, where $\beta_{1}$ is a large positive constant, shown as a rectangle in Figure 3.1. This gives meaning to the assertion 


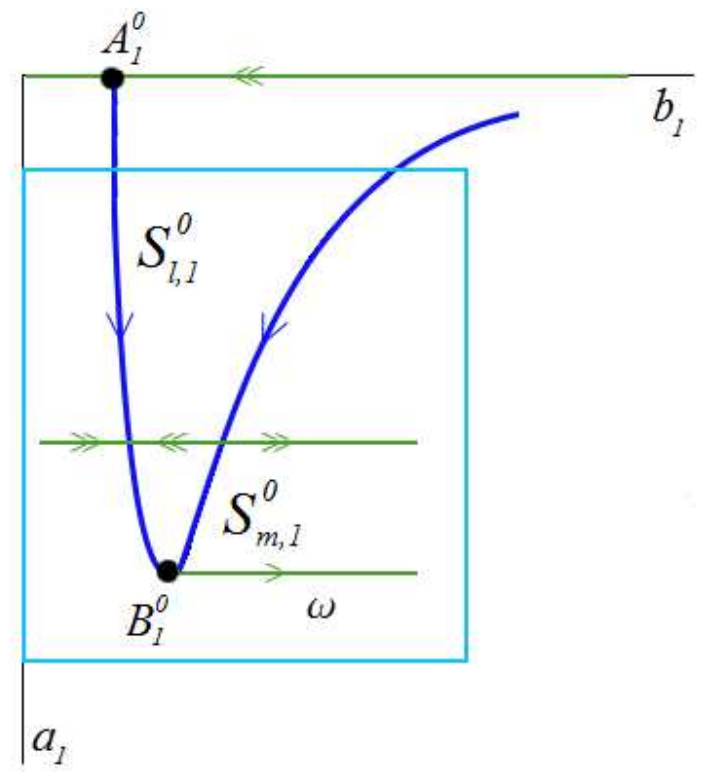

Figure 3.1. Critical manifold $S_{1}^{0}$ and fast dynamics in Regime 1.

that the original variables $a, b$ satisfy $a=O(1)$ and $b=O\left(\delta^{2}\right)$ in Regime 1 . On the domain under consideration, results for $\delta>0$ follow by regular perturbation theory, i.e. system (3.5) has a folded critical manifold $S_{1}^{\delta}$ close to $S_{1}^{0}$ with similar dynamics (slow motion on the left branch $S_{l, 1}^{\delta}$ and a fast jump from the point $B_{1}^{\delta}$ to the right). We conclude from the results described in Appendix $A$ that the attracting critical manifolds $S_{l, 1}^{0}$ and $S_{l, 1}^{\delta}$ perturb smoothly to attracting slow invariant manifolds for small $\varepsilon>0$.

REMARK 3.1. The slow manifold $S_{1}^{0}$ is the limit of parts of the rescaled slow manifolds $S_{l}^{\delta}$ and $S_{m}^{\delta}$ from Subsection 2.1 as $\delta \rightarrow 0$. The fold point $B_{1}^{0}$ corresponds to the fold point $B^{\delta}$ and the point $A_{1}^{0}=(0,1)$ corresponds to the point $A^{\delta}$. In some sense the fold point $D^{\delta}$ corresponds to $b_{1}=\infty$ on $S_{m, 1}^{0}$ in the limit $\delta \rightarrow 0$. Hence, the jump back from $D^{\delta}$ to $A^{\delta}$ in Figure 2.1 corresponds to the unbounded stable fiber of the point $A_{1}^{0}$ on the positive $b_{1}$-axis, which is however not included in Regime 1 since perturbation methods do not apply there uniformly in $\varepsilon$ and $\delta$.

3.2. Regime $2, a=O(1), b=O(1)$. In Regime 2 we consider system (2.4) away from the non-hyperbolic lines $l_{a}$ and $l_{b}$, i.e. we consider $a \in\left[\alpha_{2}, 1\right]$ and $b \in\left[\beta_{2}, 2\right]$ with constants $\alpha_{2}>0, \beta_{2}>0$. Setting $\varepsilon=0$ in system (2.4) gives the layer problem (2.6). In this regime systems (2.4) and (2.6) depend regularly in $\delta$. For $\delta=0$ the layer problem (2.6) has the form

$$
\begin{aligned}
& a^{\prime}=0 \\
& b^{\prime}=a^{2} b^{2}(1-b) .
\end{aligned}
$$

In the region under consideration the line $l_{h, 2}$ defined by $b=1$ is a normally hyperbolic attracting critical manifold. The reduced flow for system $(2.7)$ on $l_{h, 2}$ for $\delta=0$ is governed by the equation

$$
\dot{a}=a^{2}(\mu-1)
$$


hence $a$ decreases under the slow flow on $l_{h, 2}$.

For $\delta>0$ the line $l_{h, 2}$ perturbs regularly to a part of the normally hyperbolic attracting critical manifold $S_{r}^{\delta}$ with similar dynamics. Results for $\varepsilon>0$ follow from standard Fenichel theory uniformly for small $\delta$, i.e. solutions starting on the left side of $l_{h, 2}$ are rapidly attracted by the slow manifold corresponding to $l_{h, 2}$ respectively $S_{r}^{\delta}$ and follow the slow flow.

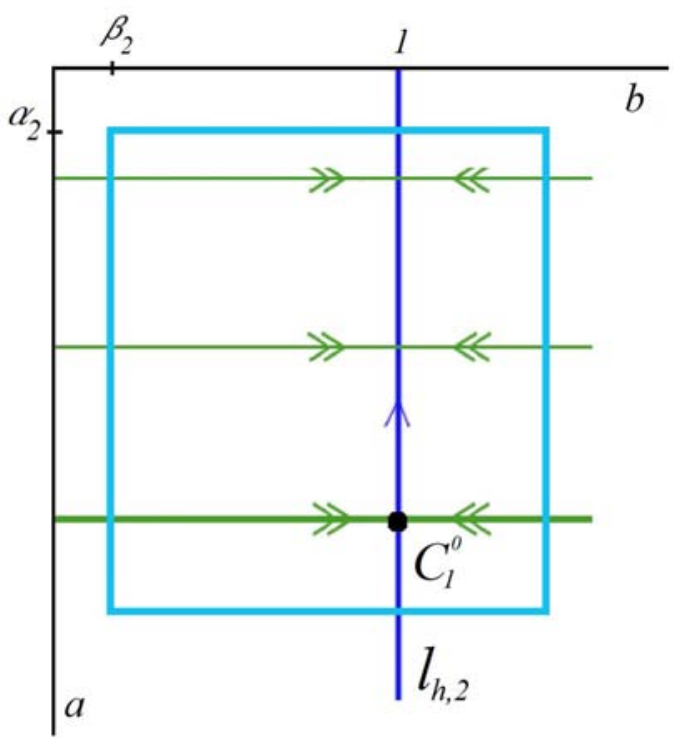

Figure 3.2. Critical manifold $l_{h, 2}$ and fast dynamics in Regime 2.

REMARK 3.2. In the limit $\delta \rightarrow 0$ the line $l_{h, 2}$ corresponds to the part of $S_{r}^{\delta}$ where the variable $a$ is large. The point $C_{1}^{0}=(1 / 2,1) \in l_{h, 2}$ corresponds to the point $C^{\delta} \in S_{r}^{\delta}$. The fold point $D^{\delta}$ is not visible in this regime. Parts of the singular orbit connecting $B_{1}^{0}$ to $C_{1}^{0}$ are visible as the left side of the stable fiber of the point $C_{1}^{0}$.

3.3. Regime 3, $a=O(\delta), b=O(1)$. We use equation (3.1) and rewrite system $(2.4)$ as

$$
\begin{aligned}
a^{\prime} & =\tilde{\varepsilon} \delta\left[a^{2} b^{2}(\mu-1)+\mu \delta^{2}\right], \\
b^{\prime} & =a^{2} b^{2}(1-b)+\delta^{2}\left(a^{2} b^{2}-b+\delta^{2}\right),
\end{aligned}
$$

where $\tilde{\varepsilon}$ causes the slow-fast structure, while $\delta$ affects mainly the shape of the critical manifold $S^{\delta}$ corresponding to $\tilde{\varepsilon}=0$.

We use the scaling

$$
a=\delta a_{3}, \quad b=b_{3}
$$

with $a_{3} \in\left[0, \alpha_{3}\right]$ and $b_{3} \in[-0.1,2]$, with a large constant $\alpha_{3}>0$. In these variables system (2.4) has the form

$$
\begin{aligned}
a_{3}^{\prime} & =\tilde{\varepsilon}\left[a_{3}^{2} b_{3}^{2}(\mu-1)+\mu\right], \\
b_{3}^{\prime} & =a_{3}^{2} b_{3}^{2}\left(1-b_{3}\right)-b_{3}+\delta\left(a_{3}^{2} b_{3}^{2}+1\right),
\end{aligned}
$$


where we have again divided out a factor $\delta$, i.e. the derivative is now with respect to the rescaled time variable $\delta \tau$. In the following system (3.10) is considered as a slowfast system with $\tilde{\varepsilon}$ being a singular perturbation parameter, whereas $\delta$ acts as a regular perturbation parameter. This is the place, where the condition (1.11) expressed in the form (3.1) is crucial. For $\delta=0$ the layer problem, obtained from (3.10) by setting $\tilde{\varepsilon}=0$ has the form

$$
\begin{aligned}
& a_{3}^{\prime}=0 \\
& b_{3}^{\prime}=a_{3}^{2} b_{3}^{2}\left(1-b_{3}\right)-b_{3} .
\end{aligned}
$$

The critical manifold $S_{3}^{0}$ of (3.11) is defined by the equation

$$
b_{3}\left[a_{3}^{2} b_{3}\left(1-b_{3}\right)-1\right]=0 .
$$

It consists of an attracting branch $S_{l, 3}^{0}$ defined by $b_{3}=0$, a repelling branch $S_{m, 3}^{0}$ and an attracting branch $S_{r, 3}^{0}$ which are the left and the right branches of the curve

$$
a_{3}=\sqrt{\frac{1}{b_{3}\left(1-b_{3}\right)}}
$$

corresponding to $b_{3} \in(0,1 / 2)$ and $b_{3} \in(1 / 2,1)$, respectively. These branches are separated by the fold point $D_{3}^{0}=(2,1 / 2)$ corresponding to the point $D^{\delta}$, see Figure 3.3 .

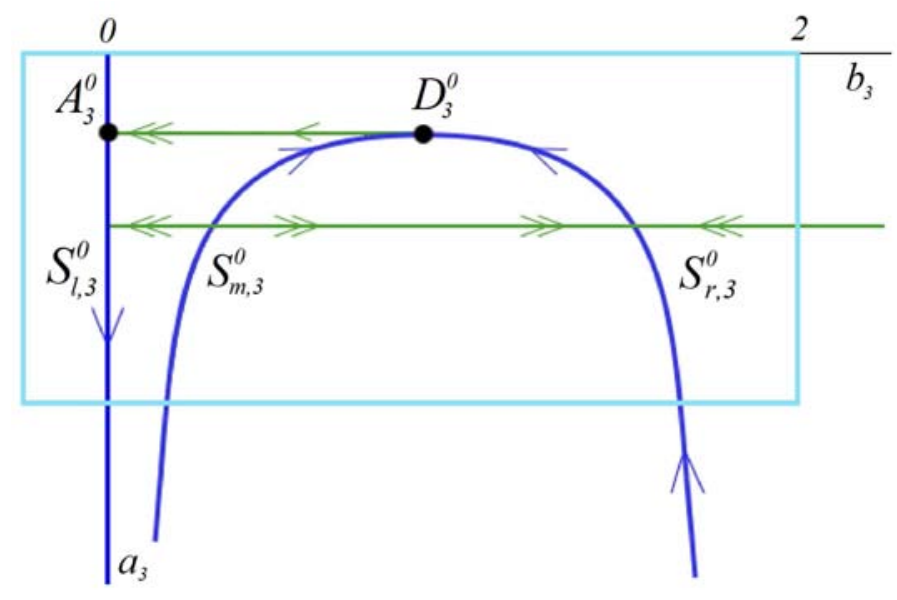

Figure 3.3. Critical manifold $S_{3}^{0}$ and fast dynamics in Regime 3.

It is easy to see that $D_{3}^{0}$ is a jump point as described in Appendix A. Our main interest is in the singular orbit which follows the slow flow on $S_{r, 3}^{0}$ until it reaches the fold point $D_{3}^{0}$ from where it jumps to $A_{3}^{0}$ to follow the slow flow on $S_{l, 3}^{0}$. Note that the fast jump from $B_{3}^{0}$ to $C_{3}^{0}$ is not visible in this regime. Since $\delta$ acts as a regular perturbation parameter in (3.10), we obtain a slightly perturbed critical manifold $S_{3}^{\delta}$ with branches $S_{l, 3}^{\delta}, S_{m, 3}^{\delta}, S_{r, 3}^{\delta}$ and the fold point $D_{3}^{\delta}$, which is still a jump point. As before these critical manifolds correspond to parts of the critical manifolds $S_{l}^{\delta}, S_{m}^{\delta}$, and $S_{r}^{\delta}$ from Subsection 2.2.

Geometric singular perturbation theory is applicable in Regime 3 to obtain rigorous results for small $\tilde{\varepsilon}>0$ uniformly in $\delta \geq 0$. In particular, we conclude from the results described in Appendix $A$ that the attracting critical manifolds $S_{r, 3}^{0}$ and $S_{r, 3}^{\delta}$ perturb smoothly to attracting slow invariant manifolds for small $\varepsilon>0$. 
3.4. Singular cycles . Based on these results we now define the singular cycle $\Gamma_{0}^{0}$ of system $(2.4)$ for $(\varepsilon, \delta)=(0,0)$ as

$$
\Gamma_{0}^{0}:=\sigma_{1} \cup \sigma_{2} \cup \sigma_{3} \cup \sigma_{4},
$$

where $\sigma_{1}$ is the segment of the slow manifold $l_{h}$ connecting the points $C^{0}=(1 / 2,1)$ to $D^{0}=(0,1), \sigma_{2}$ is the segment of the slow manifold $l_{b}$ connecting the point $D^{0}$ with the point $A^{0}=(0,0), \sigma_{3}$ is the segment of the slow manifold $l_{a}$ connecting the points $A^{0}$ to $B^{0}=(1 / 2,0)$, and $\sigma_{4}$ is the heteroclinic orbit of (2.6) connecting the point $B^{0}$ with the point $C^{0}$. Keep in mind that all of Regime 1 collapses onto the non-hyperbolic line $l_{a}$, all of Regime 3 collapses onto the non-hyperbolic line $l_{b}$, and $\sigma_{1}, \sigma_{2}, \sigma_{3}$ are just sets of equilibria of system (2.8).

The analysis in Regimes 1-3 suggests that $\Gamma_{0}^{0}$ is indeed a good candidate to obtain relaxation oscillations of the form described in Section 2 given approximately by slow motion from the point $A^{\delta}$ to $B^{\delta}$, a fast jump from $B^{\delta}$ to $C^{\delta}$, slow motion from $C^{\delta}$ to $D^{\delta}$, and a final jump from $D^{\delta}$ to $A^{\delta}$ for $(\tilde{\varepsilon}, \delta)$ close to $(0,0)$. The degeneracy of the

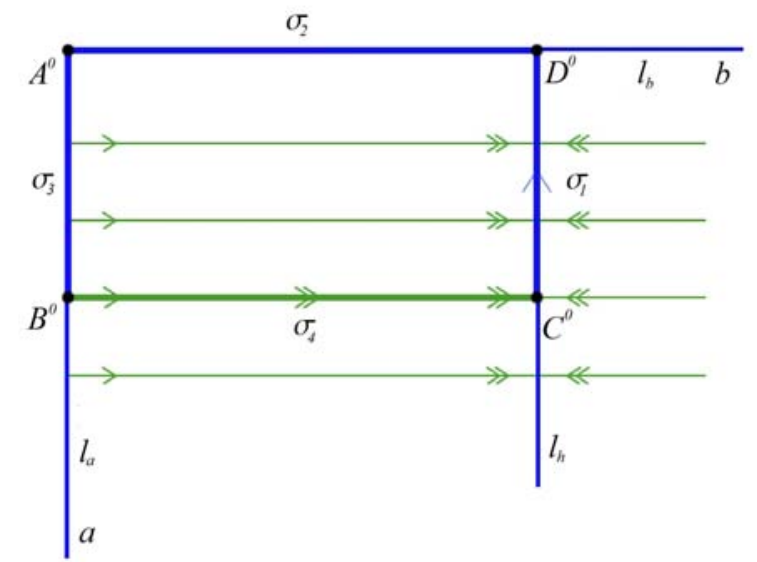

Figure 3.4. Critical manifold $S^{0}$ of system (2.6) and singular cycle $\Gamma_{0}^{0}$.

singular cycle $\Gamma_{0}^{0}$ and of the non-hyperbolic slow manifolds $l_{a}$ and $l_{b}$ has been partially resolved by the scaling methods used in Regimes 1 and 2, respectively. However, uniform perturbations results are only possible for Regimes 1-3 in the way they were defined. Note that the domains in the $(a, b)$-plane corresponding to Regimes 1-3 do not overlap. Hence, the perturbation analysis of the singular cycle $\Gamma_{0}^{0}$ requires a detailed study of extending and matching the individual regimes.

This could be possibly carried out in the framework of classical matched asymptotic expansions, but the procedure would be much more complicated than in the case of classical relaxation oscillations. Thus, the main purpose of this paper is to show that a geometric approach based on the blow-up method is well-suited to carry out the matching and to provide a comprehensive and clear picture of the global situation in this two-parameter singular perturbation problem.

Our main result is

THEOREM 3.1. For $\mu<1$ there exist $\delta_{0}>0$ and $\tilde{\varepsilon}_{0}>0$ such that system (1.9) has a unique attracting periodic orbit $\Gamma_{\varepsilon}^{\delta}$ for $0<\delta \leq \delta_{0}$ and $0<\varepsilon \leq \tilde{\varepsilon}_{0} \delta$ with the properties 
1. $\Gamma_{\varepsilon}^{\delta}$ tends to $\Gamma_{0}^{\delta}$ as $\varepsilon \rightarrow 0$ for $\delta \in\left(0, \delta_{0}\right]$,

2. $\Gamma_{\varepsilon}^{\delta}$ tends to the singular cycle $\Gamma_{0}^{0}$ as $(\delta, \varepsilon) \rightarrow(0,0)$.

Proof. The assertions of the theorem will follow from Theorem 4.10 by setting $\varepsilon=\tilde{\varepsilon} \delta$ and by applying the blow-up transformations (4.2) and (4.13).

4. Blow-up analysis. In this section we carry out the blow-up analysis for (1.9). We start by rewriting system (1.9) with $\varepsilon=\tilde{\varepsilon} \delta$ (see equation (3.1)) as

$$
\begin{aligned}
& a^{\prime}=\tilde{\varepsilon} \delta\left[a^{2} b^{2}(\mu-1)+\mu \delta^{2}\right], \\
& b^{\prime}=a^{2} b^{2}(1-b)+\delta^{2}\left(a^{2} b^{2}-b+\delta^{2}\right), \\
& \delta^{\prime}=0,
\end{aligned}
$$

i.e. we consider (4.1) as a three-dimensional vector field $X_{\tilde{\varepsilon}}$ defined on $\mathbb{R}^{3}$, by treating the parameter $\delta$ as a variable, while $\tilde{\varepsilon}$ is the singular perturbation parameter causing the slow-fast structure. Obviously, all planes $\delta=$ const. are invariant for system (4.1). The family of critical manifolds $S^{\delta}, \delta \in\left[0, \delta_{0}\right]$, from Section 2.1 and Section 3 is now viewed as a two-dimensional critical manifold $S$. For $\delta>0$ the critical manifold $S$ has a folded structure, i.e. $S=S_{l} \cup S_{m} \cup S_{r}$ with folds along the curves $F_{B}:=\left\{\left(B^{\delta}, \delta\right): \delta \in\left[0, \delta_{0}\right]\right\}$ and $F_{D}:=\left\{\left(D^{\delta}, \delta\right): \delta \in\left[0, \delta_{0}\right]\right\}$. On the plane $\delta=0$ the critical manifold $S$ limits on $S^{0} \times\{0\}$ with $S^{0}=l_{a} \cup l_{b} \cup l_{h}$. For $\delta$ bounded away from zero the results on the existence of slow manifolds and relaxation cycles from Section 2.2 can be readily interpreted for analogous two dimensional objects obtained by adding the $\delta$ direction.

It turns out that the main task is to analyze the dynamics close to the degenerate lines $l_{a} \cup\{0\}$ and $l_{b} \cup\{0\}$ where for $\tilde{\varepsilon}=0$ the linearization of system (4.1) at points of $l_{a} \cup\{0\}$ and $l_{b} \cup\{0\}$ has a triple zero eigenvalue. This will be achieved by a cylindrical blow-up of the line $l_{b} \cup\{0\}$ followed by a cylindrical blow-up of the line $l_{a} \cup\{0\}$, which leads to a desingularization of the extended system (4.1) at $\delta=0$. Roughly speaking the non-hyperbolic lines $l_{a}$ and $l_{b}$ will be blown-up to cylinders by introducing suitable polar-like coordinates in the directions transverse to the lines. We will see that the blow-up procedure is able to resolve the degeneracies of the original problem. In particular, the critical manifold $S$ of system (4.1) will be blown-up to a critical manifold $\bar{S}$, which is normally hyperbolic away from the fold curves.

The analysis of the blown-up problem will be carried out in charts $K_{1}-K_{4}$ introduced below. We use the following notation: any object $O$ of the extended system (4.1) is denoted as $\bar{O}$ for the blown-up problem, and by $M_{i}$ in chart $K_{i}, i=1, \ldots, 4$. It will turn out that the charts $K_{1}$ and $K_{3}$ correspond to the scaling Regimes 1 and 3 , respectively, and that scaling Regime 2 is covered by parts of chart $K_{2}$ (and also by parts of $K_{4}$ ). We will see that chart $K_{2}$ and parts of $K_{4}$ provide sufficient overlap to match the Regimes 1-3. We will be able to identify a singular cycle $\bar{\Gamma}_{0}^{0}$ of the blown-up system with improved hyperbolicity and transversality properties. Once the correct singular cycle has been found the proof of Theorem 3.1 on the existence of the relaxation cycle can be based on well established methods from geometric singular perturbation theory.

4.1. Blow-up of the non-hyperbolic line $l_{b} \times\{0\}$. We define the blow-up transformation of the non-hyperbolic line of steady states $l_{b} \times\{0\}$ by

$$
\begin{aligned}
a & =r \bar{a} \\
b & =\bar{b} \\
\delta & =r \bar{\delta}
\end{aligned}
$$


with $(\bar{a}, \bar{\delta}, r, \bar{b}) \in \mathbb{S}^{1} \times \mathbb{R} \times \mathbb{R}$. The line $l_{b} \times\{0\}$ corresponding to $r=0$ is blown-up to the cylinder $Z_{b}:=\mathbb{S}^{1} \times\{0\} \times \mathbb{R}$, see Figure 4.1.

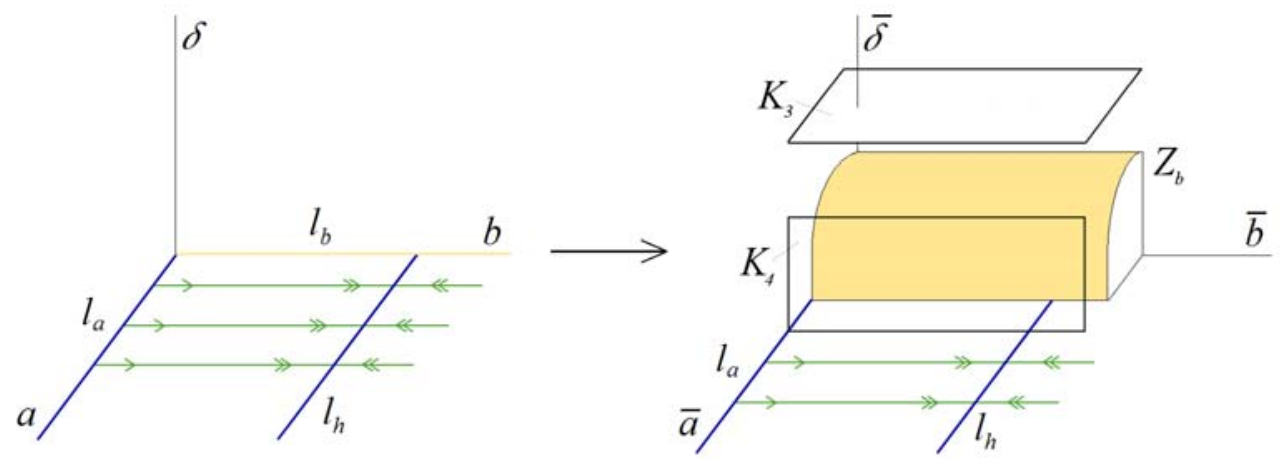

Figure 4.1. Blow-up of the non-hyperbolic line $l_{b}$.

The vector field (4.1) induces a vector field on the blown-up space $\mathbb{S}^{1} \times \mathbb{R} \times \mathbb{R}$. Since the cylinder $Z_{b}$ is constructed as the blow-up of a line of equilibria, the blown-up vector field vanishes on the cylinder. To obtain a non-trivial flow on the cylinder the blown-up vector field must be divided by a suitable power of $r$. Note that $\delta=0$ in the original system corresponds to either $\bar{\delta}=0$ or $r=0$, thus the dynamics of the blown-up system in the plane $\bar{\delta}=0$ and on the cylinder $Z_{b}$ is particularly important.

The blown-up vector field is analyzed in charts $K_{3}, K_{4}$ defined by setting $\bar{\delta}=1$, $\bar{a}=1$, respectively, in the blow-up transformation (4.2), again see Figure 4.1. Thus, chart $K_{3}$ covers the whole upper part of the cylinder corresponding to $\bar{\delta}>0$, while $K_{4}$ covers its front side corresponding to $\bar{a}>0$. In chart $K_{3}$ the blow-up transformation is given by

$$
a=r_{3} a_{3}, \quad b=b_{3}, \quad \delta=r_{3} .
$$

and in chart $K_{4}$ the blow-up transformation is given by

$$
a=r_{4}, \quad b=b_{4}, \quad \delta=r_{4} \delta_{4} .
$$

Dynamics in $K_{3}$. Since $r_{3}=\delta$ the transformation (4.3) is precisely the scaling transformation (3.9) used in Regime 3. After dividing out a factor $r_{3}$ system (4.1) written in $K_{3}$ has the form

$$
\begin{aligned}
a_{3}^{\prime} & =\tilde{\varepsilon}\left[a_{3}^{2} b_{3}^{2}(\mu-1)+\mu\right], \\
b_{3}^{\prime} & =a_{3}^{2} b_{3}^{2}\left(1-b_{3}\right)-b_{3}+r_{3}\left(a_{3}^{2} b_{3}^{2}+1\right) \\
r_{3}^{\prime} & =0
\end{aligned}
$$

where ' denotes the derivative with respect to the rescaled time scale $r_{3} \tau$. System (4.5) is the same as system (3.10), i.e. $K_{3}$ corresponds to Regime 3 . In this chart the cylinder $Z_{b}$ corresponds to the plane $r_{3}=0$. Thus, the geometric singular perturbation analysis of Regime 3 with respect to $\tilde{\varepsilon}$ is valid on compact domains. The relevant dynamics on the cylinder are as shown in Figure 3.3. 
Dynamics in $K_{4}$. Inserting (4.4) into system (4.1) gives a system of differential equations for $r_{4}, b_{4}, \delta_{4}$. We obtain

$$
\begin{aligned}
r_{4}^{\prime} & =\tilde{\varepsilon} r_{4}^{3} \delta_{4}\left[b_{4}^{2}(\mu-1)+\mu \delta_{4}^{2}\right], \\
b_{4}^{\prime} & =r_{4}^{2}\left[b_{4}^{2}\left(1-b_{4}\right)+\delta_{4}^{2}\left(r_{4}^{2} b_{4}^{2}-b_{4}+r_{4}^{2} \delta_{4}^{2}\right)\right], \\
\delta_{4}^{\prime} & =-\tilde{\varepsilon} r_{4}^{2} \delta_{4}^{2}\left[b_{4}^{2}(\mu-1)+\mu \delta_{4}^{2}\right] .
\end{aligned}
$$

By dividing out a factor $r_{4}^{2}$ we obtain the (partially desingularized) blown-up system

$$
\begin{aligned}
r_{4}^{\prime} & =\tilde{\varepsilon} r_{4} \delta_{4}\left[b_{4}^{2}(\mu-1)+\mu \delta_{4}^{2}\right], \\
b_{4}^{\prime} & =b_{4}^{2}\left(1-b_{4}\right)+\delta_{4}^{2}\left(r_{4}^{2} b_{4}^{2}-b_{4}+r_{4}^{2} \delta_{4}^{2}\right), \\
\delta_{4}^{\prime} & =-\tilde{\varepsilon} \delta_{4}^{2}\left[b_{4}^{2}(\mu-1)+\mu \delta_{4}^{2}\right] .
\end{aligned}
$$

The derivative in (4.7) is with respect to a rescaled time variable $\tau_{4}$. The plane $r_{4}=0$, which corresponds to the cylinder $Z_{b}$, and the plane $\delta_{4}=0$, are both invariant under the flow of (4.7). System (4.7) is a singularly perturbed system with slow variables $r_{4}, \delta_{4}$, fast variable $b_{4}$ and singular perturbation parameter $\tilde{\varepsilon}$.

Setting $\tilde{\varepsilon}=0$ gives the layer problem

$$
\begin{aligned}
& r_{4}^{\prime}=0, \\
& b_{4}^{\prime}=b_{4}^{2}\left(1-b_{4}\right)+\delta_{4}^{2}\left(r_{4}^{2} b_{4}^{2}-b_{4}+r_{4}^{2} \delta_{4}^{2}\right), \\
& \delta_{4}^{\prime}=0 .
\end{aligned}
$$

The surface defined by

$$
b_{4}^{2}\left(1-b_{4}\right)+\delta_{4}^{2}\left(r_{4}^{2} b_{4}^{2}-b_{4}+r_{4}^{2} \delta_{4}^{2}\right)=0
$$

is denoted by $S_{4}$, since it corresponds to the critical manifold $S$ of system (4.1). Instead of analyzing the equation defining $S_{4}$ directly, it is instructive to restrict attention to the invariant planes $r_{4}=0$ and $\delta_{4}=0$ of system (4.7).

In the invariant plane $r_{4}=0$ the dynamics is governed by

$$
\begin{aligned}
& b_{4}^{\prime}=b_{4}^{2}\left(1-b_{4}\right)-\delta_{4}^{2} b_{4}, \\
& \delta_{4}^{\prime}=-\tilde{\varepsilon} \delta_{4}^{2}\left[b_{4}^{2}(\mu-1)+\mu \delta_{4}\right] .
\end{aligned}
$$

This system is the standard form of slow-fast systems with respect to the small parameter $\tilde{\varepsilon}$, i.e. with slow variable $\delta_{4}$ and fast variable $b_{4}$. Setting $\tilde{\varepsilon}=0$ gives the layer problem

$$
\begin{aligned}
& b_{4}^{\prime}=b_{4}^{2}\left(1-b_{4}\right)-\delta_{4}^{2} b_{4} \\
& \delta_{4}^{\prime}
\end{aligned}
$$

The critical manifold $\hat{S}_{4}$ of system (4.11), defined by the equation

$$
b_{4}\left[b_{4}\left(1-b_{4}\right)-\delta_{4}^{2}\right]=0
$$

consists of the invariant line $b_{4}=0$, denoted by $\hat{S}_{l, 4}$, a branch $\hat{S}_{m, 4}$ and a branch $\hat{S}_{4, r}$, which are given by

$$
\delta_{4}=\sqrt{b_{4}\left(1-b_{4}\right)}
$$

with $b_{4} \in[0,1 / 2)$ and $b_{4} \in(1 / 2,1]$, respectively, see Figure 4.2 . Obviously, $\hat{S}_{l, 4}, \hat{S}_{m, 4}$, $\hat{S}_{r, 4}$ are the branches of the intersection of $S_{4}$ with the plane $r_{4}=0 . \hat{S}_{m, 4}$ and $\hat{S}_{r, 4}$ 
are separated by the fold point $D_{4}^{0}=(1 / 2,1 / 2) . \hat{S}_{l, 4}$ and $\hat{S}_{m, 4}$ intersect in the nonhyperbolic point $(0,0)$. The branch $\hat{S}_{r, 4}$ is attracting for the flow in $r_{4}=0$. Similarly, away from the origin the line $\hat{S}_{l, 4}$ is attracting, while the branch $\hat{S}_{m, 4}$ is repelling. The variable $\delta_{4}$ increases for the slow flow on $\hat{S}_{r, 4}$, while it decreases for the slow flow on $\hat{S}_{l, 4}$. Hence the fold point $D_{4}^{0}$ is a jump point from where a fast jump to the point $A_{4}^{0}=(0,1 / 2) \in \hat{S}_{l, 4}$ takes place. Note that the origin is still a degenerate steady state of system (4.11).

REMARK 4.1. The relevant dynamics in chart $K_{3}$ takes place in the region $a_{3} \geq 1$. Since this region is covered by chart $K_{4}$, chart $K_{3}$ is not explicitly needed in our analysis, i.e. the critical manifold $S_{3}^{0}$ corresponds to the parts of $\hat{S}_{4}$ with $\delta_{4} \geq 1 / \alpha_{3}$ (compare Figure 3.1 with Figure 4.2). Furthermore, the parts of $\hat{S}_{4}$ with $\delta_{4} \in\left[0,1 / \alpha_{3}\right]$ correspond to the unbounded branches of $S_{3}^{0}$ corresponding to $a_{3} \geq \alpha_{3}$ which had to be excluded from the analysis in Regime 3.

REMARK 4.2. It is an important property of the blow-up method that all results, which are obtained in the scaling regimes, are recovered in some charts, i.e. here in chart $K_{3}$. The power of the blow-up method comes from the fact that other charts, i.e. here chart $K_{4}$, provide a compactification of the unbounded domains in the scaling regimes where perturbation methods were not applicable.

In the invariant plane $\delta_{4}=0$ the dynamics is governed by

$$
\begin{aligned}
r_{4}^{\prime} & =0 \\
b_{4}^{\prime} & =b_{4}^{2}\left(1-b_{4}\right) .
\end{aligned}
$$

The equilibria of this system are the line $b_{4}=0$ and the line $b_{4}=1$, which we denote by $l_{a, 4}$ and $\check{S}_{r, 4}$, respectively. Clearly, $l_{a, 4}$ and $\check{S}_{r, 4}$ are the intersections of $S_{4}$ with the plane $\delta_{4}=0$. The curves $\hat{S}_{r, 4}$ and $\check{S}_{r, 4}$ meet at the point $\check{q}_{4}=(0,1,0)$. Similarly, the curves $\hat{S}_{l, 4}, \hat{S}_{m, 4}$ and $l_{a, 4}$ intersect in the origin, see Figure 4.2 .

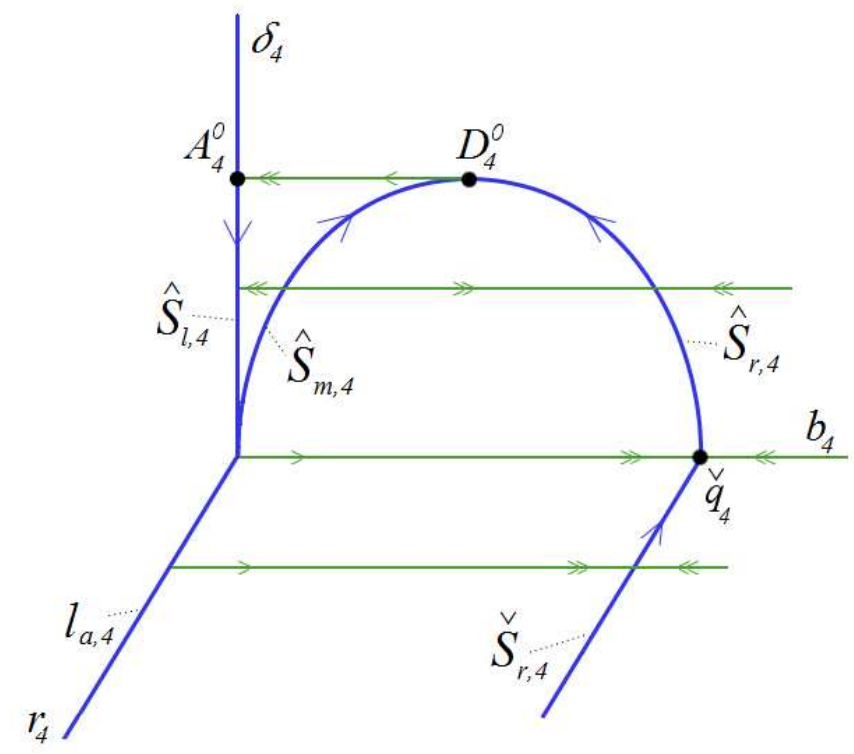

Figure 4.2. Dynamics of system (4.7) in $r_{4}=0$ and $\delta_{4}=0$.

From the above discussion we conclude 
LEMmA 4.1. The critical manifold $S_{4}$ described by the equation (4.9) has the following properties at least for $r_{4}$ or $\delta_{4}$ small:

1. $S_{4}$ is smooth away from the line $l_{a, 4}$.

2. $S_{4}$ has a folded structure, i.e. $S_{4}=S_{l, 4} \cup S_{m, 4} \cup S_{r, 4}$, where $S_{m, 4}$ and $S_{r, 4}$ are separated by the fold curve $F_{D, 4}$ while the branches $S_{l, 4}$ and $S_{m, 4}$ intersect cusp-like along $l_{a, 4}$. The branches $S_{l, 4}$ and $S_{r, 4}$ are attracting and $S_{m, 4}$ is repelling for the layer problem (4.8).

3. The branch $S_{r, 4}$ limits on $\hat{S}_{r, 4}$ for $r_{4}=0$ and on $\check{S}_{r, 4}$ for $\delta_{4}=0$. Similarly, $S_{m, 4}$ limits on $\hat{S}_{m, 4}$ for $r_{4}=0$ and on $l_{a, 4}$ for $\delta_{4}=0$. $S_{l, 4}$ limits on $\hat{S}_{l, 4}$ for $r_{4}=0$ and on $l_{a, 4}$ for $\delta_{4}=0$. The fold curve $F_{D, 4}$ limits on $D_{4}^{0}$ (see Figure 4.2).

Proof. Outside a neighborhood of the line $l_{a, 4}$ the assertions of the lemma follow from the implicit function theorem and the structural stability of folds. The properties of the critical manifold $S_{4}$ close to the line $l_{a, 4}$ will be proven in Subsection 4.2.

Summing up, the singular relaxation cycle $\Gamma_{0,4}^{0}$ in chart $K_{4}$ consists of slow motion along the branch $\check{S}_{r, 4}$, followed by slow motion along $\hat{S}_{r, 4}$ from the point $\check{q}_{4}$ to the fold point $D_{4}^{0}$, a fast jump from the point $D_{4}^{0}$ to the point $A_{4}^{0}$, slow motion along the branch $\hat{S}_{l, 4}$ from the point $A_{4}^{0}$ to the origin, followed by (not yet analyzed) slow motion along the line $l_{a, 4}$, see Figure 4.2. Since the linearization of system (4.7) at points in the line $l_{a, 4}$ has a triple zero eigenvalue for $\tilde{\varepsilon}=0$, the line $l_{a, 4}$ is still degenerate. The geometry of the critical manifold $S_{4}$ and the dynamics in a neighborhood of the line $l_{a, 4}$ will be studied by means of a further blow-up in the following.

4.2. Blow-up of the non-hyperbolic line $l_{a, 4}$. To study the dynamics of system (4.7) close to the non-hyperbolic line $l_{a, 4}$, we introduce the blow-up transformation

$$
\begin{aligned}
& r_{4}=\bar{r}, \\
& b_{4}=\rho^{2} \bar{b}, \\
& \delta_{4}=\rho \bar{\delta}
\end{aligned}
$$

with $(\bar{b}, \bar{\delta}, \rho, \bar{r}) \in \mathbb{S}^{1} \times \mathbb{R} \times \mathbb{R}$. By this construction the line $l_{a, 4}$ is blown-up to the cylinder $Z_{a}:=\mathbb{S}^{1} \times\{0\} \times \mathbb{R}$, see Figure 4.3. The vector field (4.7) induces a vector field on the blown-up space $\mathbb{S}^{1} \times \mathbb{R} \times \mathbb{R}$, which again leaves the cylinder $Z_{a}$ invariant. The analysis of this blown-up vector field is performed in local charts $K_{1}, K_{2}$, which are defined by setting $\bar{\delta}=1, \bar{b}=1$, respectively, in the blow-up transformation (4.13). Chart $K_{1}$ covers the upper part of the cylinder $Z_{a}$ corresponding to $\bar{\delta}>0$, while $K_{2}$ covers its front side corresponding to $\bar{b}>0$. In chart $K_{1}$ the blow-up transformation is given by

$$
r_{4}=r_{1}, \quad b_{4}=\rho_{1}^{2} b_{1}, \quad \delta_{4}=\rho_{1},
$$

and in chart $K_{2}$ the blow-up transformation is given by

$$
r_{4}=r_{2}, \quad b_{4}=\rho_{2}^{2}, \quad \delta_{4}=\rho_{2} \delta_{2} .
$$

Dynamics in $K_{1}$. By inserting the transformation (4.14) into system (4.7) and 


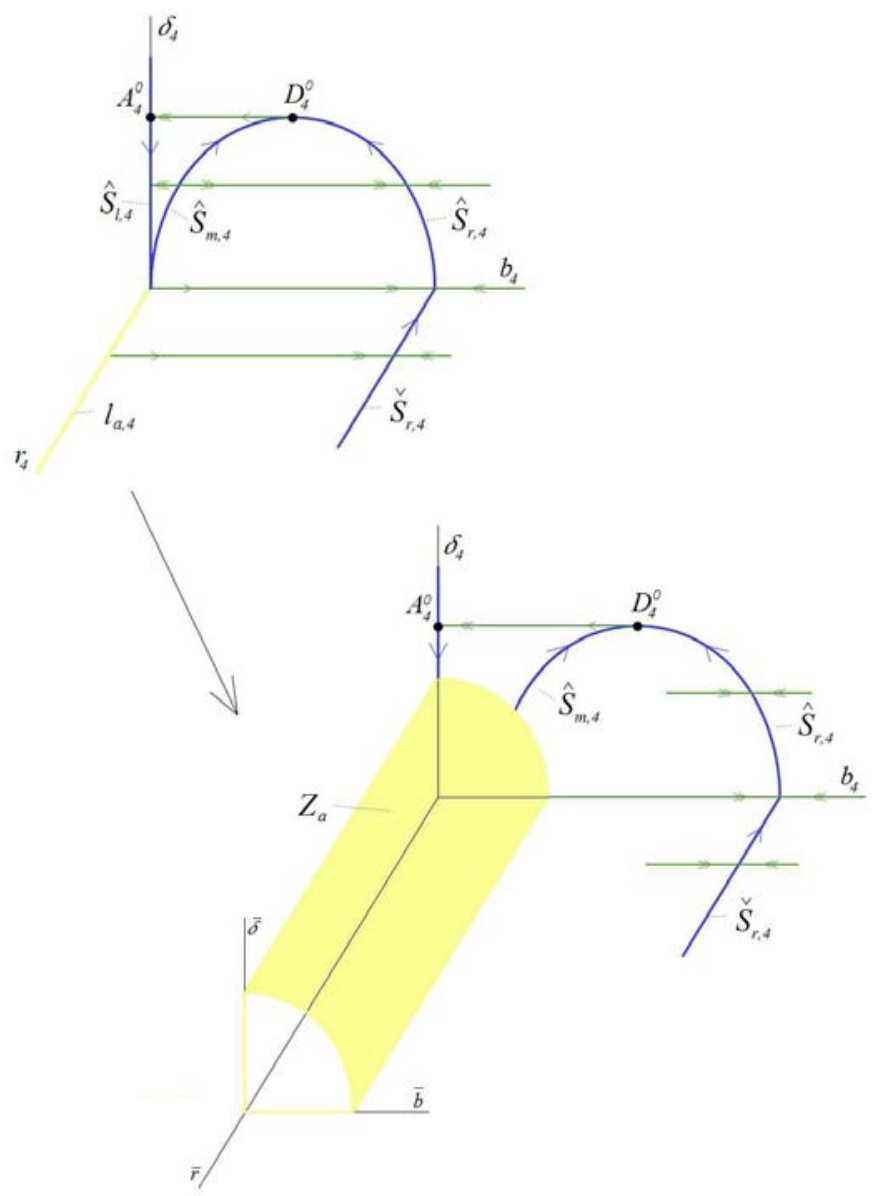

Figure 4.3. Blow-up transformation (4.13) for system (4.7).

dividing out a factor of $\rho_{1}^{2}$ we obtain the final desingularized blown-up system

$$
\begin{aligned}
& r_{1}^{\prime}=\tilde{\varepsilon} r_{1} \rho_{1}\left[\rho_{1}^{2} b_{1}^{2}(\mu-1)+\mu\right], \\
& b_{1}^{\prime}=2 \tilde{\varepsilon} \rho_{1}\left[\rho_{1}^{2} b_{1}^{2}(\mu-1)+\mu\right]+b_{1}^{2}\left(1-\rho_{1}^{2} b_{1}\right)+\rho_{1}^{2} r_{1}^{2} b_{1}^{2}-b_{1}+r_{1}^{2}, \\
& \rho_{1}^{\prime}=-\tilde{\varepsilon} \rho_{1}^{2}\left[\rho_{1}^{2} b_{1}^{2}(\mu-1+\mu)\right] .
\end{aligned}
$$

System (4.16) is a singularly perturbed system with slow variables $r_{1}, \rho_{1}$, fast variable $b_{1}$, singular perturbation parameter $\tilde{\varepsilon}$, and the layer problem

$$
\begin{aligned}
& r_{1}^{\prime}=0, \\
& b_{1}^{\prime}=b_{1}^{2}\left(1-\rho_{1}^{2} b_{1}\right)+\rho_{1}^{2} r_{1}^{2} b_{1}^{2}-b_{1}+r_{1}^{2}, \\
& \rho_{1}^{\prime}=0 .
\end{aligned}
$$

The layer problem (4.17) has a two-dimensional critical manifold $S_{1}$ described by the equation

$$
b_{1}^{2}\left(1-\rho_{1}^{2} b_{1}\right)+\rho_{1}^{2} r_{1}^{2} b_{1}^{2}-b_{1}+r_{1}^{2}=0 .
$$


As before, we first restrict attention to the invariant planes, namely the plane $r_{1}=0$ which corresponds to a region in the front of the cylinder $Z_{b}$ of the first blow-up and the plane $\rho_{1}=0$ corresponding to the cylinder $Z_{a}$.

In the invariant plane $r_{1}=0$ the dynamics is governed by

$$
\begin{aligned}
b_{1}^{\prime} & =2 \tilde{\varepsilon} \rho_{1}\left[\rho_{1}^{2} b_{1}^{2}(\mu-1)+\mu\right]+b_{1}^{2}\left(1-\rho_{1}^{2} b_{1}\right)-b_{1}, \\
\rho_{1}^{\prime} & =-\tilde{\varepsilon} \rho_{1}^{2}\left[\rho_{1}^{2} b_{1}^{2}(\mu-1+\mu)\right]
\end{aligned}
$$

which is a slow-fast system with singular perturbation parameter $\tilde{\varepsilon}$, fast variable $b_{1}$ and slow variable $\rho_{1}$. The corresponding layer problem $(\tilde{\varepsilon}=0)$ has the form

$$
\begin{aligned}
& b_{1}^{\prime}=b_{1}^{2}\left(1-\rho_{1}^{2} b_{1}\right)-b_{1}, \\
& \rho_{1}^{\prime}=0 .
\end{aligned}
$$

The corresponding critical manifold $\hat{S}_{1}$ defined by the equation $b_{1}\left[b_{1}\left(1-\rho_{1}^{2} b_{1}\right)-1\right]=0$ consists of a stable branch $\hat{S}_{l, 1}$ with $b_{1}=0$, an unstable branch $\hat{S}_{m, 1}$ and a stable branch $\hat{S}_{r, 1}$ given by $\rho_{1}=\sqrt{\frac{1}{b_{1}}-\frac{1}{b_{1}^{2}}}$ with $b_{1} \in[1,2)$ and $b_{1} \in(2, \infty)$, respectively. The fold point $D_{1}^{0}=(2,1 / 2)$ separates the branches $\hat{S}_{m, 1}$ and $\hat{S}_{r, 1}$, see Figure 4.4. Again, $\hat{S}_{l, 1}, \hat{S}_{m, 1}$ and $\hat{S}_{r, 1}$ are the branches of the intersection of $S_{1}$ with the plane $r_{1}=0$.

REMARK 4.3. The configuration in the plane $r_{1}=0$ of chart $K_{1}$ is a desingularized version of the configuration in the plane $r_{4}=0$ of chart $K_{4}$. The degenerate equilibrium at the origin of system (4.11) has been blown-up to the line $\rho_{1}=0$ (which is in fact the circle $\mathbb{S}^{1} \times\{0\} \times\{0\}$ on the cylinder of the second blow-up). The branches $\hat{S}_{l, 1}$ and $\hat{S}_{m, 1}$ which intersected in chart $K_{4}$ have been separated and are attracting and repelling, respectively. Their endpoints in the plane $r_{1}=0$ are denoted by $\hat{p}_{1}=(0,0)$ and $\hat{q}_{1}=(1,0)$.

In the invariant plane $\rho_{1}=0$ system $(4.16)$ reduces to

$$
\begin{aligned}
r_{1}^{\prime} & =0, \\
b_{1}^{\prime} & =b_{1}^{2}-b_{1}+r_{1}^{2} .
\end{aligned}
$$

The equilibria of system (4.21) are given by the curve $r_{1}=\sqrt{b_{1}-b_{1}^{2}}$. This curve, denoted by $\check{S}_{1}$, consists of a stable branch $\check{S}_{l, 1}$ corresponding to $b_{1} \in[0,1 / 2)$, a repelling branch $\check{S}_{m, 1}$ corresponding to $b_{1} \in(1 / 2,1]$, and the fold point $B_{1}^{0}=(1 / 2,1 / 2)$, see Figure 4.4. Clearly, $\check{S}_{l, 1}$ and $\check{S}_{m, 1}$ are the branches of the intersection of $S_{1}$ with the plane $\rho_{1}=0$.

REMARK 4.4. Chart $K_{1}$ covers essentially Regime 1. In particular, the manifold $\check{S}_{1}$ described above is precisely the critical manifold $S_{1}^{0}$ of system (3.4) from Regime 1.

REMARK 4.5. Note that for $\rho_{1}=0$ the reduced (slow) vector fields with respect to $\tilde{\varepsilon}$ on $\check{S}_{l, 1}$ and $\check{S}_{m, 1}$ (on the corresponding slow manifolds) vanish identically. Hence, the fold point $B_{1}^{0}$ is not a jump point in the sense of Appendix A. This apparent difficulty is in some sense an artefact caused by setting $\varepsilon=\tilde{\varepsilon} \delta$ which is needed in Regime 3 but not in Regime 1, see also the proof of Lemma 4.8.

As before we conclude

LEMMA 4.2. The critical manifold $S_{1}$ defined by the equation (4.18) has the following properties at least for $r_{1}$ or $\rho_{1}$ small:

1. $S_{1}$ is smooth.

2. $S_{1}$ has a folded structure, i.e. $S_{1}=S_{l, 1} \cup S_{m, 1} \cup S_{r, 1}$, where $S_{l, 1}$ and $S_{r, 1}$ are attracting and $S_{m, 1}$ is repelling for the layer problem (4.17). The branches 


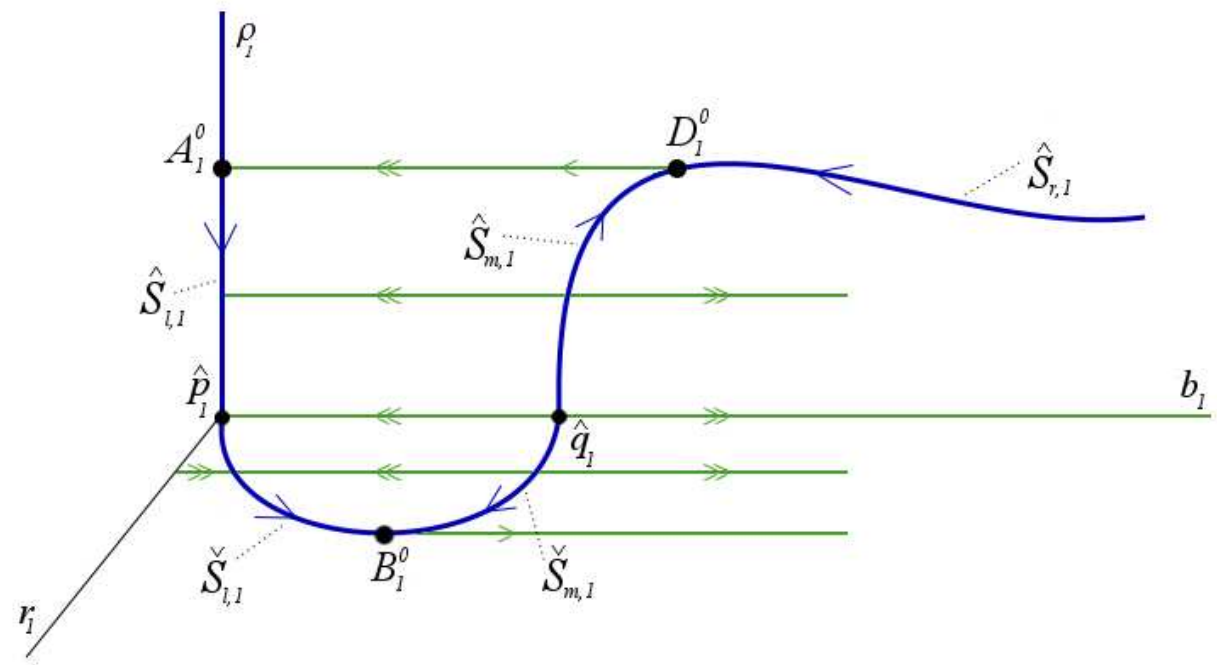

Figure 4.4. Dynamics in $K_{1}$.

$S_{l, 1}$ and $S_{m, 1}$ are separated by the fold curve $F_{B, 1}$ while $S_{m, 1}$ and $S_{r, 1}$ are separated by the fold curve $F_{D, 1}$.

3. The branch $S_{l, 1}$ limits on $\hat{S}_{l, 1}$ for $r_{4}=0$ and on $\check{S}_{l, 1}$ for $\rho_{1}=0$. Similarly, $S_{m, 1}$ limits on $\hat{S}_{m, 1}$ for $r_{1}=0$ and on $\check{S}_{m, 1}$ for $\rho_{1}=0$. $S_{r, 1}$ limits on $\hat{S}_{r, 1}$ for $r_{1}=0$. The fold curve $F_{D, 1}$ limits on $D_{1}^{0}$ and the fold curve $F_{B, 1}$ limits on $B_{1}^{0}$ (see Figure 4.4).

Summing up, the singular relaxation cycle $\Gamma_{0,1}^{0}$ in chart $K_{1}$ follows the slow dynamics along the branch $\hat{S}_{r, 1}$ to the fold point $D_{1}^{0}$, the fast orbit from the point $D_{1}^{0}$ to the point $A_{1}^{0}$, and the branch $S_{l, 1}$ from the point $A_{1}^{0}$ to the point $B_{1}^{0}$ through the point $\hat{p}_{1}$, and finally jumps to the right from the point $B_{1}^{0}$. To see how the singular orbit continues for large $b_{1}$ we have to switch to chart $K_{2}$.

Dynamics in $K_{2}$. By inserting transformation (4.15) into system (4.7) and dividing out a factor of $\rho_{2}^{2}$ we obtain the final desingularized blown-up system written in chart $K_{2}$

$$
\begin{aligned}
r_{2}^{\prime} & =\tilde{\varepsilon} r_{2} \rho_{2} \delta_{2}\left[\rho_{2}^{2}(\mu-1)+\mu \delta_{2}^{2}\right] \\
\rho_{2}^{\prime} & =\frac{1}{2} \rho_{2}\left[1-\rho_{2}^{2}+\delta_{2}^{2}\left(r_{2}^{2} \rho_{2}^{2}-1+r_{2}^{2} \delta_{2}^{2}\right)\right], \\
\delta_{2}^{\prime} & =-\tilde{\varepsilon} \rho_{2} \delta_{2}^{2}\left[\rho_{2}^{2}(\mu-1)+\mu \delta_{2}^{2}\right]-\frac{1}{2} \delta_{2}\left[1-\rho_{2}^{2}+\delta_{2}^{2}\left(r_{2}^{2} \rho_{2}^{2}-1+r_{2}^{2} \delta_{2}^{2}\right)\right] .
\end{aligned}
$$

System (4.22) is a singularly perturbed system with singular perturbation parameter $\tilde{\varepsilon}$. Setting $\tilde{\varepsilon}=0$ gives the layer problem

$$
\begin{aligned}
r_{2}^{\prime} & =0 \\
\rho_{2}^{\prime} & =\frac{1}{2} \rho_{2}\left[1-\rho_{2}^{2}+\delta_{2}^{2}\left(r_{2}^{2} \rho_{2}^{2}-1+r_{2}^{2} \delta_{2}^{2}\right)\right] \\
\delta_{2}^{\prime} & =-\frac{1}{2} \delta_{2}\left[1-\rho_{2}^{2}+\delta_{2}^{2}\left(r_{2}^{2} \rho_{2}^{2}-1+r_{2}^{2} \delta_{2}^{2}\right)\right] .
\end{aligned}
$$

The layer problem (4.23) has a 2-dimensional critical manifold $S_{2}$ described by the 
equation

$$
1-\rho_{2}^{2}+\delta_{2}^{2}\left(r_{2}^{2} \rho_{2}^{2}-1+r_{2}^{2} \delta_{2}^{2}\right)=0
$$

Additionally, there exists a line of equilibria $l_{2}$ defined by $\delta_{2}=0, \rho_{2}=0$, i.e. $l_{2}$ is the $r_{2}$-axis.

REMARK 4.6. All of the critical manifold $S_{2}$ has been covered in chart $K_{1}$ and $K_{4}$ already. The following discussion of $S_{2}$ is included just for completeness. The important dynamics occurs close to the line $l_{2}$ which is not visible in other charts and provides the so far missing overlap between chart $K_{1}$ and $K_{4}$ (i.e. Regime 1 and Regime 3).

REMARK 4.7. Note that away from $S_{2}, \rho_{2}$ and $\delta_{2}$ are fast variables. This shows that system (4.22) is not in the standard form (1.1) of slow-fast systems. However, Fenichel Theory applies in this more general situation as well. Since we focus on the dynamics close to the line $l_{2}$ we do not give further details.

The planes $r_{2}=0, \rho_{2}=0$, and $\delta_{2}=0$ are invariant under the flow of (4.22). The plane $r_{2}=0$ corresponds to a region in the front of the cylinder $Z_{b}$ of the first blow-up, while the plane $\rho_{2}=0$ corresponds to the front side of the cylinder $Z_{a}$ of the second blow-up, see Figure 4.5.

In the invariant plane $\rho_{2}=0$ system (4.22) reduces to the system

$$
\begin{aligned}
& r_{2}^{\prime}=0 \\
& \delta_{2}^{\prime}=-\frac{1}{2} \delta_{2}\left[1+r_{2}^{2} \delta_{2}^{4}-\delta_{2}^{2}\right]
\end{aligned}
$$

The line $l_{2}$ is attracting for this system. Again, the equation $1+r_{2}^{2} \delta_{2}^{4}-\delta_{2}^{2}=0$ defines a folded curve $\check{S}_{2}$ of equilibria. The fold point $B_{2}^{0}$ separates $\check{S}_{2}$ into an attracting branch $\check{S}_{l, 2}$, and a repelling branch $\check{S}_{m, 2}$, see Figure 4.5 . Clearly, $\check{S}_{l, 2}$ corresponds to the curve $\breve{S}_{l, 1}$ and $\check{S}_{m, 2}$ corresponds to the curve $\check{S}_{m, 1}$ from chart $K_{1}$.

In the invariant plane $\delta_{2}=0$ system (4.22) has the form

$$
\begin{aligned}
\rho_{2}^{\prime} & =\frac{1}{2} \rho_{2}\left(1-\rho_{2}^{2}\right) \\
r_{2}^{\prime} & =0
\end{aligned}
$$

The line $\check{S}_{r, 2}$ defined by $\rho_{2}=1$ and the line $l_{2}$ are the lines of equilibria. The situation in the plane $\delta_{2}=0$ is similar to the situation in the plane $\delta_{4}=0$ in chart $K_{4}$, with the only difference that the non-hyperbolic line $l_{a, 4}$ from chart $K_{4}$ has been replaced by the hyperbolic line $l_{2}$ due to the second blow-up (4.13). Within $\delta_{2}=0$ the line $l_{2}$ is repelling and the line $\check{S}_{r, 2}$ is attracting.

The dynamics in the plane $r_{2}=0$ can be analyzed similarly and turns out to be consistent with results obtained in charts $K_{1}$ and $K_{4}$. Since this part of phase space is already covered by charts $K_{1}$ and $K_{4}$, we omit more details. We find a curve of equilibria connecting the point $\check{q}_{2}$ to the point $\hat{q}_{2}$, which consists of an attracting branch $\hat{S}_{r, 2}$ and a repelling branch $\hat{S}_{m, 2}$ separated by a fold point $D_{2}^{0}$, see Figure 4.5 .

As before we conclude

LEMMA 4.3. The critical manifold $S_{2}$ defined by equation (4.24) has the following properties if at least one of the variables $r_{2}, \rho_{2}$, or $\delta_{2}$ is small:

1. $S_{2}$ is smooth.

2. $S_{2}$ is a folded surface, i.e. $S_{2}=S_{l, 2} \cup S_{m, 2} \cup S_{r, 2}$ with fold curves $F_{B, 2}$ and $F_{D, 2}$, where $S_{l, 2}$ and $S_{m, 2}$ are attracting and $S_{r, 2}$ is repelling for the layer problem (4.23). 


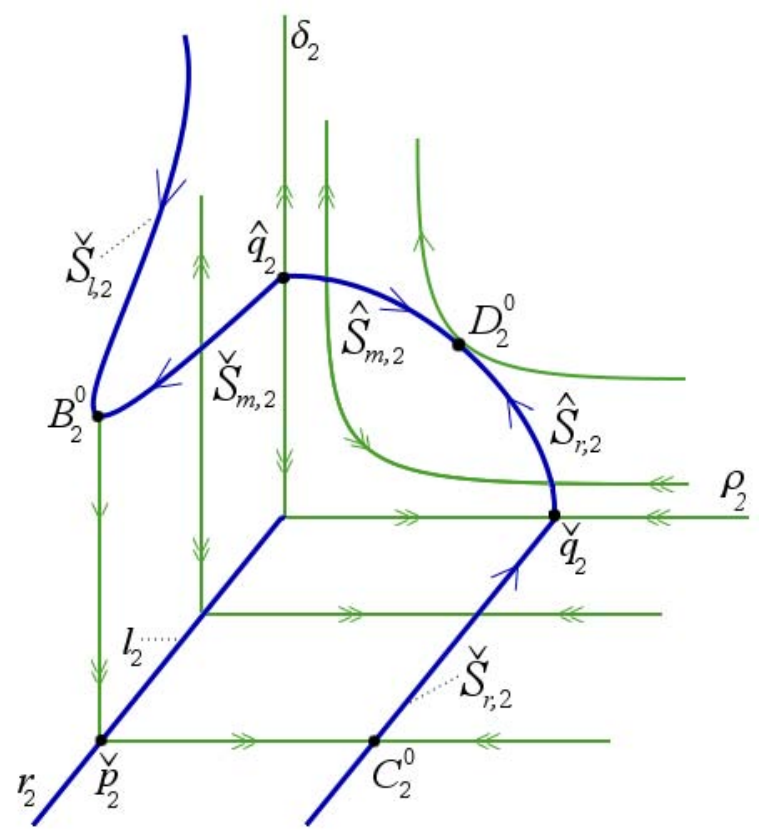

Figure 4.5. Dynamics in $K_{2}$.

3. The branch $S_{l, 2}$ limits on $\check{S}_{l, 2}$ for $\rho_{2}=0$. The branch $S_{m, 2}$ limits on $\check{S}_{m, 2}$ for $\rho_{2}=0$ and on $\hat{S}_{l, 2}$ for $r_{2}=0$. Similarly, $S_{r, 2}$ limits on $\hat{S}_{r, 2}$ for $r_{2}=0$ and on $\breve{S}_{r, 2}$ for $\delta_{2}=0$. The fold curve $F_{B, 2}$ limits on $B_{2}^{0}$ and $F_{D, 2}$ limits on $D_{2}^{0}$ (see Figure 4.5).

LEMMA 4.4. The line $l_{2}$ considered as a line of equilibria of system (4.22) is of saddle-type. Its stable manifold lies in the plane $\rho_{2}=0$ and its unstable manifold lies in the plane $r_{2}=0$.

Summing up, we obtain the following description of the singular relaxation cycle in chart $K_{2}$. From the fold point $B_{2}^{0}$ there is a fast jump to the point $\check{p}_{2}=(1 / 2,0,0) \in l_{2}$, then a fast jump to the point $C_{2}^{0}=(1 / 2,1,0)$, followed by slow motion along $\check{S}_{r, 2}$ to the point $D_{2}^{0}$ from where another fast jump takes place.

4.3. Properties of the blown-up system. The geometry of the blown-up space $\bar{M}$ after the two consecutive blow-ups (4.2) and (4.13) is shown schematically in Figure 4.6. Recall that first the line $l_{b}$ is blown-up to the cylinder $Z_{b}$ shown in the back of the figure. In the second blow-up (4.13) the line $l_{a}$ (actually a part of its preimage under the first blow-up) is blown-up to the cylinder $Z_{a}$, shown in the front of the figure. The vector field $X_{\tilde{\varepsilon}}$ on $M=\mathbb{R}^{3}$ corresponding to system (4.1) induces the blown-up vector field $\bar{X}_{\tilde{\varepsilon}}$ on the blown-up space $\bar{M}$, which has been analyzed in detail in the individual charts. Recall that the blow-up construction resolves the degeneracy of the critical manifold $S$ at $\delta=0$, while $\tilde{\varepsilon}$ still acts as a singular perturbation parameter in the vector field $\bar{X}_{\tilde{\varepsilon}}$. The equation $\delta^{\prime}=0$ from system (4.1) implies that the blown-up phase space has an invariant foliation corresponding to constant values of $\delta$. The singular leaf corresponding to $\delta=0$ is the union of the cylinders $Z_{b}, Z_{a}$, and the plane $\Delta$, where the plane $\Delta$ is defined by setting $\bar{\delta}=0$ in (4.13), see Figure 4.6 . 
These properties imply that the vector field $\bar{X}_{0}$ restricted to $Z_{b} \cup Z_{a} \cup \Delta$ provides the unperturbed dynamics corresponding to $(\tilde{\varepsilon}, \delta)=(0,0)$. In particular, we will be able to identify the singular cycle $\bar{\Gamma}_{0}^{0} \subset Z_{b} \cup Z_{a} \cup \Delta$. The analysis in the individual charts $K_{4}, K_{1}$ and $K_{2}$ implies the following results, see also Figure 4.6.

THEOREM 4.5. The blown-up vector field $\bar{X}_{0}$ on $\bar{M}$ has the following properties:

1. There exists a smooth two-dimensional critical manifold $\bar{S}$ with a folded structure, i.e.

$$
\bar{S}=\bar{S}_{l} \cup \bar{F}_{B} \cup \bar{S}_{m} \cup \bar{F}_{D} \cup \bar{S}_{r},
$$

where $\bar{S}_{l}$ and $\bar{S}_{r}$ are attracting, and $\bar{S}_{m}$ is repelling. The attracting branches of $S$ are separated from the repelling branch $\bar{S}_{m}$ by the fold curves $\bar{F}_{B}$ and $\bar{F}_{D}$.

2. There exists a singular orbit $\bar{\Gamma}_{0}^{0}$ of relaxation type, defined by

$$
\bar{\Gamma}_{0}^{0}=\omega_{1} \cup \omega_{2} \cup \omega_{3} \cup \omega_{4} \cup \omega_{5} \cup \omega_{6} \cup \omega_{7}
$$

with a fast jump from $\bar{B}^{0}$ to $\check{p} \in l$ along a heteroclinic orbit $\omega_{1}$ on $Z_{a}$, a fast jump from $\check{p}$ to the point $\bar{C}^{0} \in S_{r}$ along a heteroclinic orbit $\omega_{2}$ in the plane $\Delta=0$, a segment $\omega_{3}$ of $\bar{S}_{r}$ from the point $\bar{C}^{0}$ to the point $\breve{q}$ in the plane $\delta=0$, a segment $\omega_{4}$ of $\bar{S}_{r}$ from the point $\check{q}$ to the fold point $\bar{D}^{0}$ on $Z_{b}$, a fast jump from the fold point $\bar{D}^{0}$ to the point $\bar{A}^{0} \in \bar{S}_{l}$ along a heteroclinic orbit $\omega_{5}$ on the cylinder $Z_{b}$, a segment $\omega_{6}$ of $\bar{S}_{l}$ from $\bar{A}^{0}$ to the point $\hat{p}$ on the cylinder $Z_{b}$, and a final segment $\omega_{7}$ of $\bar{S}_{l}$ from the point $\hat{p}$ to the fold point $\bar{B}^{0}$ on the cylinder $Z_{a}$.

3. Within each leaf $\delta=$ const. there exists a singular cycle $\bar{\Gamma}_{0}^{\delta}$ for $\delta \in\left(0, \delta_{0}\right]$ for $\delta_{0}$ small following the slow flow on $\bar{S}_{r}$ and $\bar{S}_{l}$ with jumps at the fold curves $\bar{F}_{B}$ and $\bar{F}_{D}$ which limits on $\bar{\Gamma}_{0}^{0}$ as $\delta \rightarrow 0$.

REMARK 4.8. The singular behavior of the folded-shaped critical manifold $S^{\delta}$ of system (2.6) for $\delta \rightarrow 0$ (with the degenerate critical manifold $S^{0}$ of system (2.8) for $\delta=0$ ) has been desingularized such that its branched structure is visible again. Intuitively, our blow-up construction prevents the non-uniform collapse of the critical manifold $S^{\delta}$ onto the degenerate manifold $S^{0}$, see Remark 2.2.

Similarly, the non-uniform behavior of the singular cycle $\Gamma_{0}^{\delta}$ for system (2.4) as $\delta \rightarrow 0$ has been resolved in the blown-up problem. In this global picture of the critical manifold $\bar{S}$ we recover all the results from the scaling Regimes 1-3. In addition, compact neighborhoods of the intersections $Z_{b} \cap \Delta, Z_{a} \cap \Delta$, and $Z_{b} \cap Z_{a}$ cover the unbounded domains of the scaling regimes which cannot be analyzed perturbatively. We will see that smoothness and hyperbolicity properties of the blown-up vector field $\bar{X}_{\tilde{\varepsilon}}, \tilde{\varepsilon} \in\left[0, \tilde{\varepsilon}_{0}\right]$ permit a perturbation analysis in these neighborhoods, which allows to match the regimes. A first result in this direction is that the attracting slow manifolds from Regimes 1-3 fit together smoothly as parts of global attracting slow manifolds.

THEOREM 4.6. There exists $\tilde{\varepsilon}_{0}>0$ such that the blown-up vector field $\bar{X}_{\tilde{\varepsilon}}$ has smooth attracting slow manifolds $\bar{S}_{l, \tilde{\varepsilon}}$ and $\bar{S}_{r, \tilde{\varepsilon}}$ for $\tilde{\varepsilon} \in\left[0, \tilde{\varepsilon}_{0}\right]$.

Proof. The result follows from Fenichel theory [4], [10] applied to normally hyperbolic parts of the critical manifolds $\bar{S}_{r}$ and $\bar{S}_{l}$.

REMARK 4.9. In the following we will assume that the slow manifolds have been extended beyond the fold lines $\bar{F}_{B}$ and $\bar{F}_{D}$ by the flow corresponding to the blown-up vector field $\bar{X}_{\tilde{\varepsilon}}$.

4.4. Poincaré map and existence of limit cycles. To prove Theorem 3.1 we analyze a Poincaré map defined in a neighborhood of the singular cycle $\bar{\Gamma}_{0}^{0}$. We will 


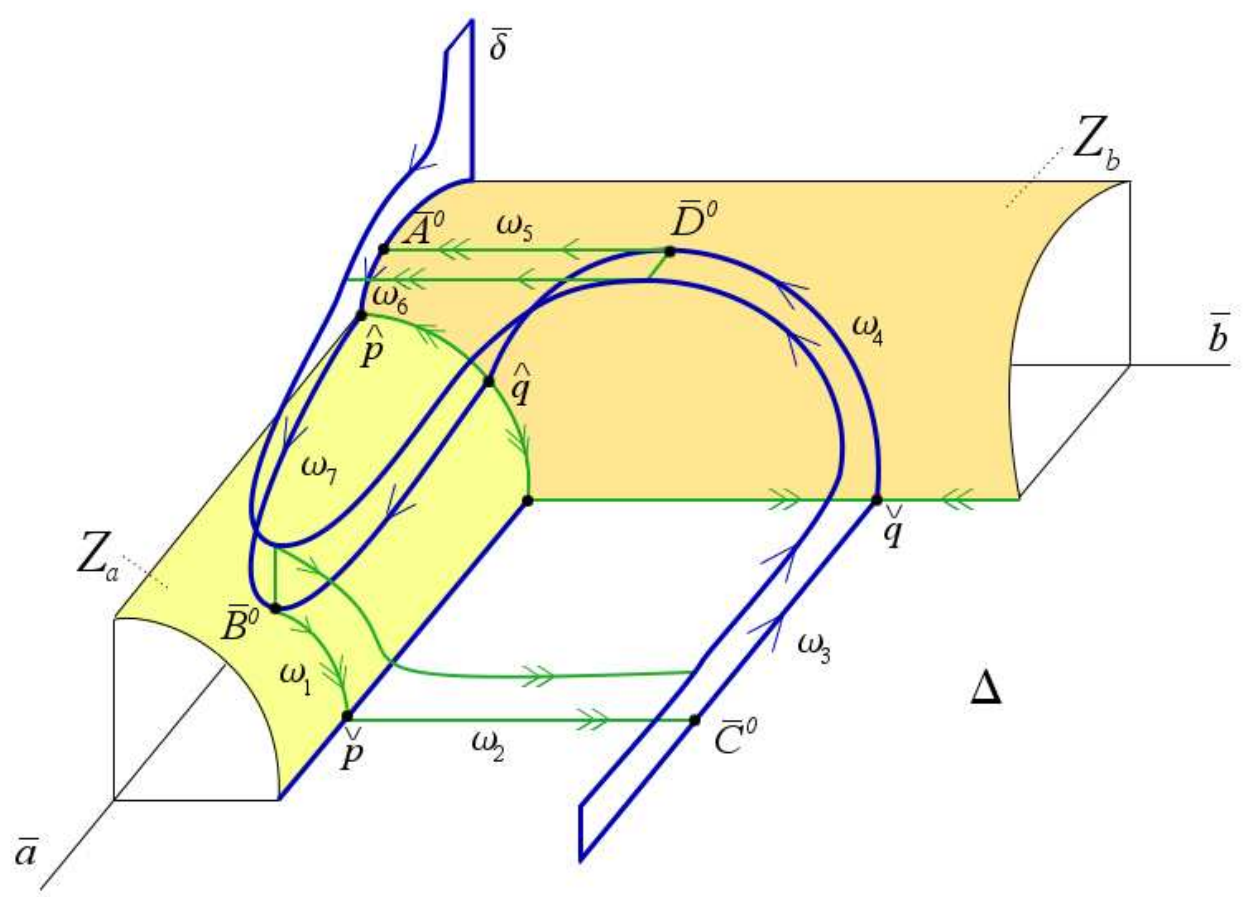

FiguRE 4.6. Geometry of the blown-up space, critical manifold $\bar{S}$ and singular cycles $\overline{\Gamma_{0}^{\delta}}$.

show that within each leaf $\delta=$ const. the Poincaré map is a strong contraction for $\tilde{\varepsilon}$ small and has an attracting fixed point corresponding to the limit cycle.

To construct the Poincaré map for the vector field $\bar{X}_{\tilde{\varepsilon}}$ we choose sections $\Sigma, \Sigma_{a}$, and $\Sigma_{b}$, as shown in Figure 4.7, i.e.

$\Sigma$ is transversal to the heteroclinic orbit $\omega_{2}$,

$\Sigma_{b}$ is transversal to the heteroclinic orbit $\omega_{5}$,

$\Sigma_{a}$ is transversal to the heteroclinic orbit $\omega_{1}$.

The sections will be defined more precisely later, where the Poincaré map is considered in the individual charts. In the following we outline the construction of the Poincaré map.

The Poincaré map will be obtained as the composition of three maps. All orbits starting in $\Sigma$ approach $\bar{S}_{r, \tilde{\varepsilon}}$, follow the slow flow along $\bar{S}_{r, \tilde{\varepsilon}}$, pass the non-hyperbolic fold curve $\bar{F}_{D}$ and follow the heteroclinic orbit $\omega_{5}$ to intersect $\Sigma_{b}$. This defines the map

$$
\Pi_{1}: \Sigma \rightarrow \Sigma_{b}
$$

Similarly, all orbits starting in $\Sigma_{b}$ approach $\bar{S}_{l, \tilde{\varepsilon}}$, follow the slow flow along $\bar{S}_{l, \tilde{\varepsilon}}$ until they pass the non-hyperbolic fold curve $\bar{F}_{B}$ and follow the heteroclinic orbit $\omega_{1}$ to intersect $\Sigma_{a}$. This defines the map

$$
\Pi_{2}: \Sigma_{b} \rightarrow \Sigma_{a} .
$$




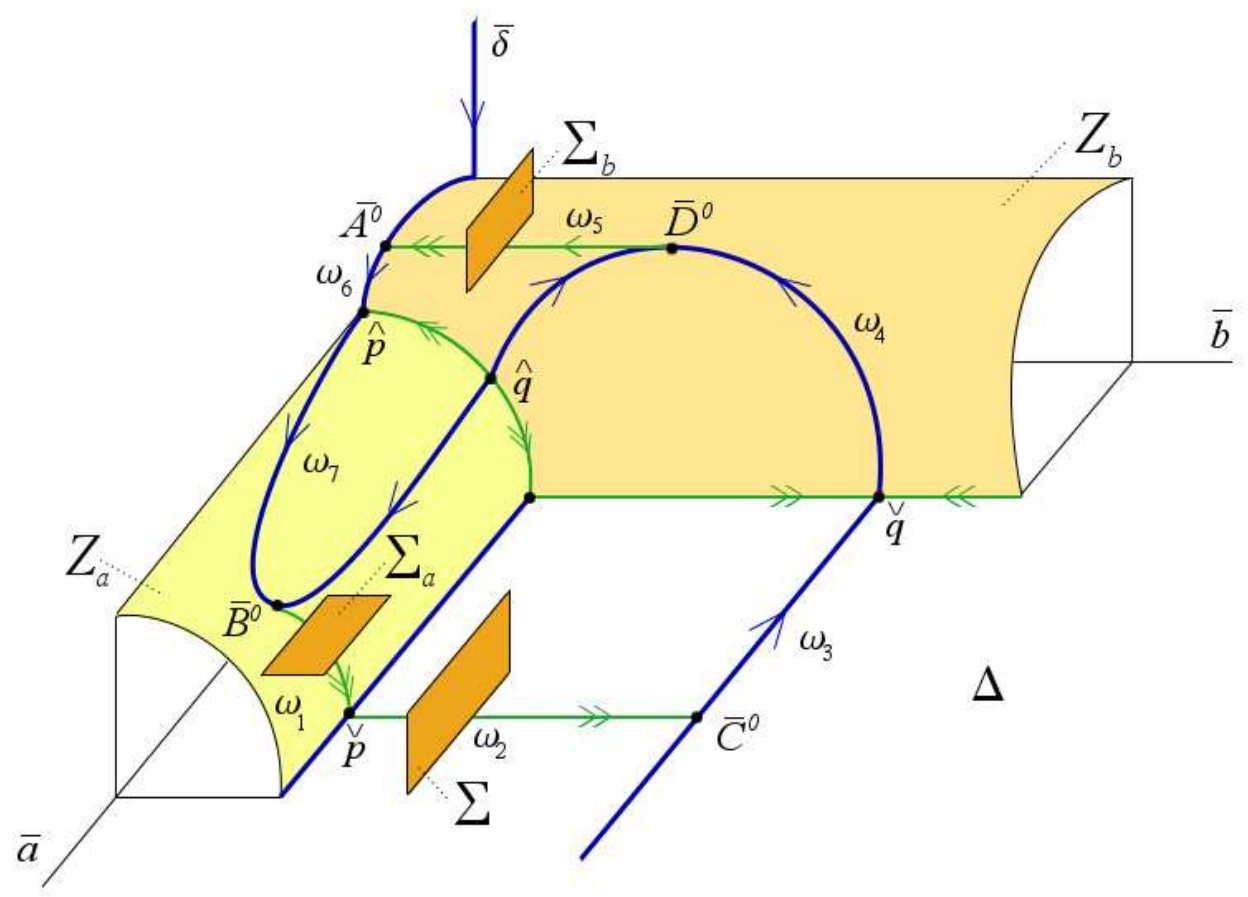

Figure 4.7. Sections for the Poincaré map.

The map

$$
\Pi_{3}: \Sigma_{a} \rightarrow \Sigma
$$

describes how orbits pass through a neighborhood of the hyperbolic line $l$. The Poincaré map $\Pi: \Sigma \rightarrow \Sigma$ is defined as

$$
\Pi=\Pi_{3} \circ \Pi_{2} \circ \Pi_{1} .
$$

The construction of the transition map $\Pi_{1}$ is carried out in chart $K_{4}$ whereas the construction of the transition maps $\Pi_{2}$ and $\Pi_{3}$ is carried out in the charts $K_{1}$ and $K_{2}$, respectively.

The transition map $\Pi_{1}$. The section $\Sigma$ is defined in chart $K_{4}$ by the conditions $b_{4}=1 / 2, r_{4}$ close to $1 / 2$, and $\left|\delta_{4}\right|$ small. Similarly, the section $\Sigma_{b}$ is defined by the conditions $b_{4}=1 / 4, \delta_{4}$ close to $1 / 2$, and $\left|r_{4}\right|$ small. Note that the invariant leaves $\delta=$ const. correspond to $\delta=\delta_{4} r_{4}$, hence these leaves are given by $\delta_{4} \approx 2 \delta$ in $\Sigma$ and $r_{4} \approx 2 \delta$ in $\Sigma_{b}$, respectively.

LEMMA 4.7. The transition map $\Pi_{1}: \Sigma \rightarrow \Sigma_{b}$ is well defined for $\tilde{\varepsilon}$ small enough. There exists a constant $c>0$ such that the map $\Pi_{1}$ restricted to a leaf $\delta=$ const. is a contraction with a contraction rate $e^{-c / \delta \tilde{\varepsilon}}$.

Proof. All orbits starting in $\Sigma$ are attracted by the extended slow manifold $\bar{S}_{r, \tilde{\varepsilon}}$ at the exponential rate stated above. The results in [13], [22] on the behavior of slow manifolds near generic folds imply that the intersection of $\bar{S}_{r, \tilde{\varepsilon}}$ with $\Sigma_{b}$ is a curve $\sigma_{4, \tilde{\varepsilon}}$ 
which is $O\left(\tilde{\varepsilon}^{2 / 3}\right)$ close to the curve given by $\bar{\Gamma}_{0}^{\delta} \cap \Sigma_{b}$ with $\delta$ small. Hence, all of $\Sigma$ is mapped to a thin wedge exponentially close to $\sigma_{4, \tilde{\varepsilon}}$.

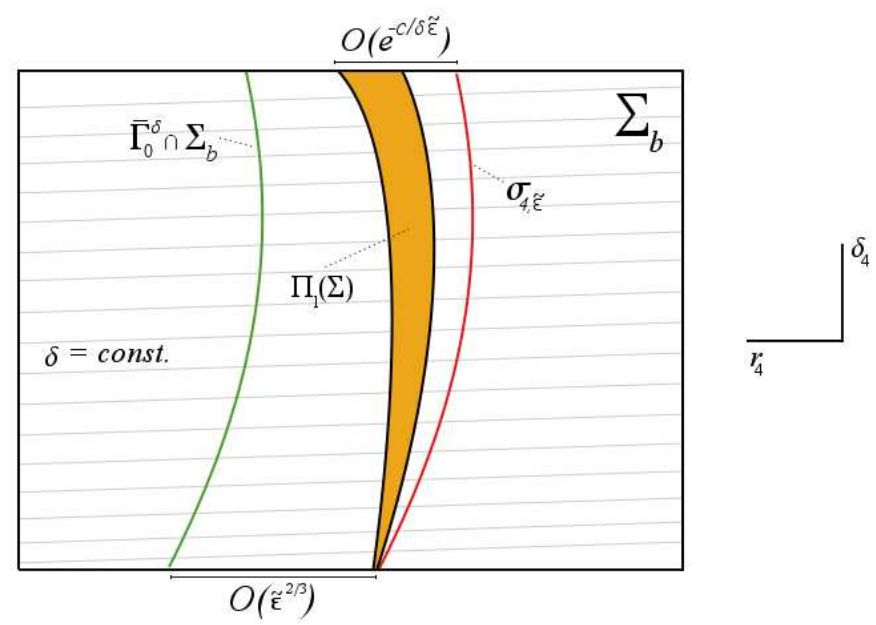

FiguRE 4.8. Image of the section $\Sigma$ under the transition map $\Pi_{1}$.

$\square$

REMARK 4.10. The above proof is carried out in chart $K_{4}$ in which the blownup vector field is three-dimensional. However, the region corresponding to the fold curve is also covered by chart $K_{3}$, i.e. Regime 3. The governing equations (3.10) in Regime 3 are a family of singularly perturbed planar vector fields depending regularly on the parameter $\delta$. Therefore, the description of the passage of the fold point can be also based on the results for the planar singularly perturbed fold in [13] which are summarized in Appendix A. Although these two points of view are equivalent, the latter is useful to obtain a more detailed description. For the analysis of the fold curve in chart $K_{4}$ one has to use the results of [22] for folds in $\mathbb{R}^{3}$.

The transition map $\Pi_{2}$. The transition map $\Pi_{2}$ is studied in chart $K_{1}$. The transformation from chart $K_{4}$ to chart $K_{1}$ is carried out according to equations (4.14). Hence, the section $\Sigma_{b}$ is now given by $\rho_{1}^{2} b_{1}=1 / 2$ with $\rho_{1}$ close to $1 / 2$, and $r_{1}$ small. Because of $\delta=\rho_{1} r_{1}$, the invariant leaves $\delta=$ const. are given by $r_{1} \approx 2 \delta$ in $\Sigma_{b}$. We define the section $\Sigma_{a}$ by $b_{1}=1, r_{1}$ close to $1 / 2$, and $\rho_{1}$ small. Because of $\delta=\rho_{1} r_{1}$, the invariant leaves $\delta=$ const. are given by $\rho_{1} \approx 2 \delta$ in $\Sigma_{a}$.

LEMMA 4.8. The transition map $\Pi_{2}: \Sigma_{b} \rightarrow \Sigma_{a}$ is well defined for $\tilde{\varepsilon}$ small enough. There exists a constant $c>0$ such that the map $\Pi_{2}$ restricted to a leaf $\delta=$ const. is a contraction with a contraction rate $e^{-c / \delta \tilde{\varepsilon}}$.

Proof. All orbits starting in $\Sigma_{b}$ are attracted by the extended slow manifold $\bar{S}_{l, \tilde{\varepsilon}}$ at the exponential rate stated above. The intersection of $\bar{S}_{l, \tilde{\varepsilon}}$ with $\Sigma_{a}$ is a curve $\sigma_{1, \tilde{\varepsilon}}$. Hence, all of $\Sigma_{b}$ is mapped to an exponentially thin wedge close to $\sigma_{1, \tilde{\varepsilon}}$. Note that the results in [13], [22] do not apply directly to system (4.16), however the dynamics close to the fold curve $F_{B, 1}$ can also be described in Regime 1, which is a family of planar singularly perturbed folds with singular perturbation parameter $\varepsilon$ parameterized by $\delta$, where the results of [13] as summarized in Appendix $A$ apply. This shows further that within a leaf $\delta=$ const. the curve $\sigma_{1, \tilde{\varepsilon}}$ is $O\left(\varepsilon^{2 / 3}\right)=O\left(\delta^{2 / 3} \tilde{\varepsilon}^{2 / 3}\right)$ close to the point $\bar{\Gamma}_{0}^{\delta} \cap \Sigma_{a}$.

口 
The transition map $\Pi_{3}$. The transition map $\Pi_{3}$ is studied in chart $K_{2}$. The transformation from chart $K_{1}$ to chart $K_{2}$ is carried out according to

$$
r_{2}=r_{1}, \quad \delta_{2}=b_{1}^{-1 / 2}, \quad \rho_{2}=b_{1}^{1 / 2} \rho_{1} .
$$

The section $\Sigma_{a}$ written in chart $K_{2}$ is given by the conditions $\delta_{2}=1, r_{2}$ close to $1 / 2$, and $\rho_{2}$ small. The invariant leaves $\delta=$ const. in $\Sigma_{a}$ are now described by $\rho_{2} \approx 2 \delta$. The transformation from chart $K_{4}$ to chart $K_{2}$ is carried out according to

$$
r_{2}=r_{4}, \quad \rho_{2}=b_{4}^{1 / 2}, \quad \delta_{2}=\delta_{4} b_{4}^{-1 / 2} .
$$

Hence, the section $\Sigma$ is now given by $\rho_{2}=1 / \sqrt{2}, r_{2}$ close to $1 / 2$ and $\delta_{2}$ small. The invariant leaves $\delta=$ const. in $\Sigma$ are now described by $\delta_{2} \approx 2 \sqrt{2} \delta$.

REMARK 4.11. In order to guarantee that $\Pi_{3}$ maps $\Sigma_{a}$ into $\Sigma$ the size of the section $\Sigma$ in the direction of $r_{2}$ (which is equal to $r_{4}$ ) has to be chosen slightly larger than the size of the section $\Sigma_{a}$ in the direction of $r_{2}$.

LEMMA 4.9. The transition map

$$
\Pi_{3}: \Sigma_{a} \rightarrow \Sigma, \quad\left(r_{2, \text { in }}, \rho_{2, \text { in }}, 1\right) \mapsto\left(r_{2, \text { out }}, 1 / \sqrt{2}, \delta_{2, \text { out }}\right)
$$

is well defined for $\tilde{\varepsilon}$ small and $\delta \in\left[0, \delta_{0}\right]$ for $\delta_{0}$ small enough. The map $\Pi_{3}$ is essentially a small translation in $r_{2}$-direction, i.e.

$$
r_{2, \text { out }}=r_{2, \text { in }}+O(\tilde{\varepsilon} \delta \ln (1 / \delta)) .
$$

Restricted to a leaf $\delta=$ const. the map is at most weakly (algebraically) expanding.

Proof. By dividing the vector field (4.22) by the non-vanishing factor

$$
\frac{1}{2} \rho_{2}\left[1-\rho_{2}^{2}+\delta_{2}^{2}\left(r_{2}^{2} \rho_{2}^{2}-1+r_{2}^{2} \rho_{2} \delta_{2}^{2}\right)\right.
$$

we rewrite the system as

$$
\begin{aligned}
r_{2}^{\prime} & =\tilde{\varepsilon} r_{2} \rho_{2} \delta_{2} R\left(r_{2}, \rho_{2}, \delta_{2}\right), \\
\rho_{2}^{\prime} & =\rho_{2}, \\
\delta_{2}^{\prime} & =-\delta_{2}+\tilde{\varepsilon} r_{2} \rho_{2} \delta_{2} R\left(r_{2}, \rho_{2}, \delta_{2}\right),
\end{aligned}
$$

with a smooth function $R\left(r_{2}, \rho_{2}, \delta_{2}\right)$ which is $O(1)$ in the domain of interest. We consider this system as a perturbation of the linear system

$$
\begin{aligned}
r_{2}^{\prime} & =0, \\
\rho_{2}^{\prime} & =\rho_{2}, \\
\delta_{2}^{\prime} & =-\delta_{2} .
\end{aligned}
$$

A short computation shows that the transition time $T$, which a solution starting in $\Sigma_{a}$ needs to reach the section $\Sigma$, is

$$
T=O\left(\ln \frac{1}{\delta}\right)
$$

where we have used that $\delta=r_{2} \rho_{2} \delta_{2}$. The assertions of the lemma follow.

This completes the construction and analysis of the Poincaré map $\Pi$ and allows to prove the existence of limit cycles for $\tilde{\varepsilon}$ small.

TheOREM 4.10. For $\mu<1$ there exists $\delta_{0}>0$ and $\tilde{\varepsilon}_{0}>0$ such that the blown-up vector field $\bar{X}_{\tilde{\varepsilon}}$ has a unique family of attracting periodic orbits $\bar{\Gamma}_{\tilde{\varepsilon}}^{\delta}$ for $0<\delta \leq \delta_{0}$ and $0<\tilde{\varepsilon} \leq \tilde{\varepsilon}_{0}$ with the properties 
1. $\bar{\Gamma}_{\tilde{\varepsilon}}^{\delta}$ tends to $\bar{\Gamma}_{0}^{\delta}$ as $\tilde{\varepsilon} \rightarrow 0$ uniformly for $\delta \in\left(0, \delta_{0}\right]$,

2. $\bar{\Gamma}_{\tilde{\varepsilon}}^{\delta}$ tends to the singular cycle $\bar{\Gamma}_{0}^{0}$ as $(\tilde{\varepsilon}, \delta) \rightarrow(0,0)$.

Proof. We conclude from Lemmas 4.7, 4.8, and 4.9 that there exist $\delta_{0}>0$ and $\tilde{\varepsilon}_{0}>0$ such that the Poincaré map $\Pi: \Sigma \rightarrow \Sigma$ is well defined for $\delta \in\left[0, \delta_{0}\right]$ and $\tilde{\varepsilon} \in\left[0, \tilde{\varepsilon}_{0}\right]$. Restricted to a leaf $\delta=$ const., i.e. $\delta=\delta_{4} r_{4}$ with $r_{4} \approx 1 / 2$, the map $\Pi$ is a contraction with contraction rate $e^{-c / \delta \tilde{\varepsilon}}$ for some constant $c>0$. The contraction mapping theorem implies the existence of a unique fixed point $\gamma_{\tilde{\varepsilon}}^{\delta}$ corresponding to the limit cycle $\bar{\Gamma}_{\tilde{\varepsilon}}^{\delta}$.

The point $\gamma_{\tilde{\varepsilon}}^{\delta}$ is $O\left(e^{-c / \delta \tilde{\varepsilon}}\right)$ close to the curve $\bar{S}_{l, \tilde{\varepsilon}} \cap \Sigma$. The proofs of Lemmas 4.8 and 4.9 imply that within a leaf $\delta=$ const. the curve $\bar{S}_{l, \tilde{\varepsilon}} \cap \Sigma$ is $O\left(\delta^{2 / 3} \tilde{\varepsilon}^{2 / 3}\right)$ close to the point $\bar{\Gamma}_{0}^{\delta} \cap \Sigma$, see Figure 4.9. The assertions of the theorem follow. $\square$

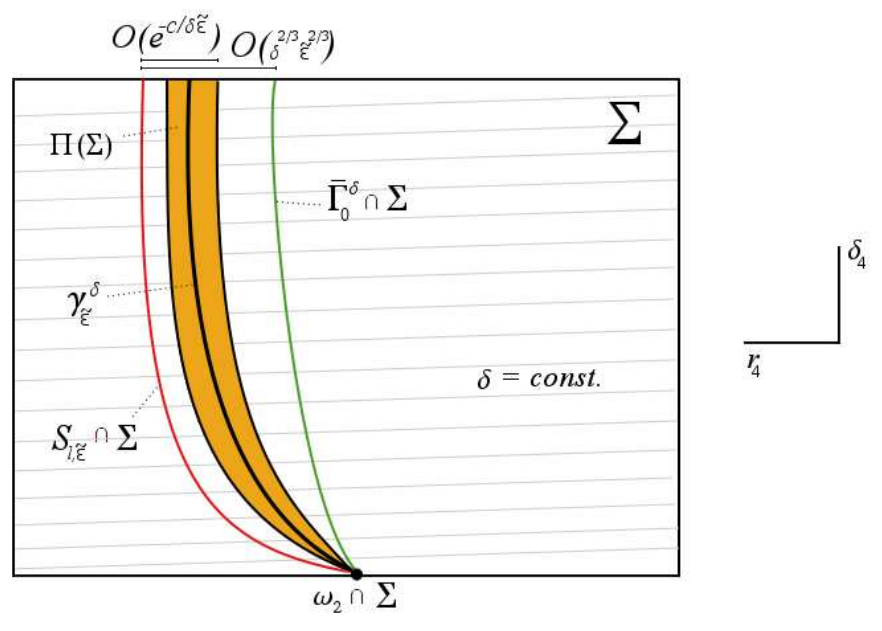

Figure 4.9. Image of the section $\Sigma$ under the Poincaré map $\Pi$.

REMARK 4.12. By setting $\varepsilon=\tilde{\varepsilon} \delta$ and by applying the blow-up transformations (4.2) and (4.13) the limit cycles $\bar{\Gamma}_{\tilde{\varepsilon}}^{\delta}$ become the limit cycles $\Gamma_{\tilde{\varepsilon}}^{\delta}$ of the original system (2.4). Thus, also the proof of Theorem 3.1 is completed.

\section{Appendix A. Slow manifolds and fold points.}

In this appendix we briefly describe results from [13] on slow manifolds of planar singularly perturbed systems with critical manifolds containing non-degenerate fold points.

We consider planar systems of the form

$$
\begin{aligned}
& x^{\prime}=f(x, y, \varepsilon), \\
& y^{\prime}=\varepsilon g(x, y, \varepsilon)
\end{aligned}
$$

with smooth functions $f$ and $g$ and a small parameter $\varepsilon>0$. The derivative in system (A.1) is with respect to the fast time scale $\tau$. By setting $\varepsilon=0$ we obtain the layer problem

$$
\begin{aligned}
& x^{\prime}=f(x, y, 0), \\
& y^{\prime}=0 .
\end{aligned}
$$


The corresponding reduced problem on the slow time scale $t=\tau \varepsilon$ is given by

$$
\begin{aligned}
& 0=f(x, y, 0) \\
& \dot{y}=g(x, y, 0) .
\end{aligned}
$$

The critical manifold $S$ is defined by the equation $f(x, y, 0)=0$. The critical manifold $S$ is a manifold of equilibria of the layer problem (A.2), while the reduced problem (A.3) is a dynamical system on $S$. Assume that the critical manifold $S$ is a folded curve with a non-degenerate fold point at the origin. Thus, $S$ consists of an attracting branch $S_{a}$ and a repelling branch $S_{r}$ with $f_{x}(x, y, 0)<0$ on $S_{a}$ and $f_{x}(x, y, 0)>0$ on $S_{r}$. At the fold point $f_{x}(0,0,0)=0$ while $f_{x x}(0,0,0) \neq 0$.

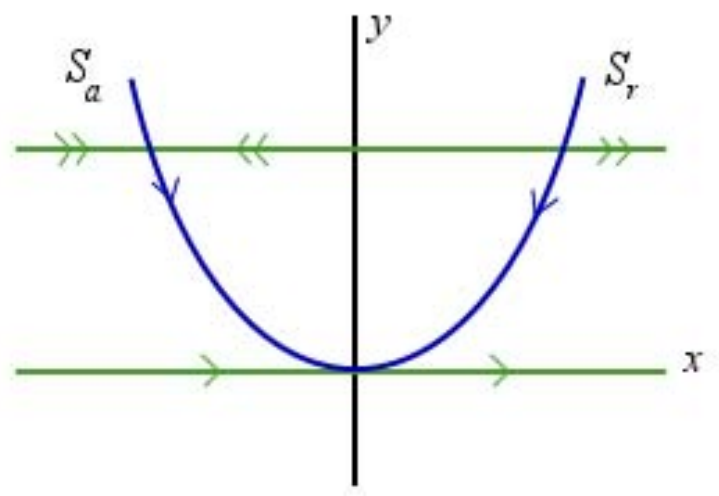

FIGURE A.1. Jump point and dynamics of the layer and the reduced problems.

Without loss of generality we assume that the position of the critical manifold $S$ is as shown in Figure A.1. We further assume that the reduced flow on $S$ is downwards, i.e. $g(x, y, 0)$ is strictly negative on $S$. The dynamics of the layer problem and the reduced problem are as shown in Figure A.1. Thus, we have the typical behavior of a jump point. Solutions starting between $S_{a}$ and $S_{r}$ are attracted by $S_{a}$, follow the reduced flow until the fold point from where they jump to the right along the weakly unstable fiber of the fold point.

Compact parts of $S_{a}$ and $S_{r}$ are normally hyperbolic. Fenichel theory [4], [10] implies that $S_{a}$ and $S_{r}$ perturb smoothly to locally invariant slow manifolds $S_{a, \varepsilon}$ and $S_{r, \varepsilon}$ for $\varepsilon$ small. The slow manifold $S_{a, \varepsilon}\left(S_{r, \varepsilon}\right)$ is attracting (repelling) and has an invariant stable (unstable) foliation with fibers close to the horizontal orbits of the layer problem. The slow flow on $S_{a, \varepsilon}$ and $S_{r, \varepsilon}$ is a smooth perturbation of the reduced flow on $S_{a}$ and $S_{r}$, hence is still directed downwards, see Figure A.2.

These results are valid outside any fixed small neighborhood of the fold point. The analysis of the asymptotic behavior of solutions close to the fold point has been the central problem in the analysis of relaxation oscillations [6], [16] by the method of matched asymptotic expansions. A more recent approach in the analysis of critical manifolds with non-hyperbolic points based on the blow-up method was introduced in [1]. A detailed geometric analysis of the dynamics and asymptotics close to the fold point based on the blow-up method is given in [13]. There it is shown that the 


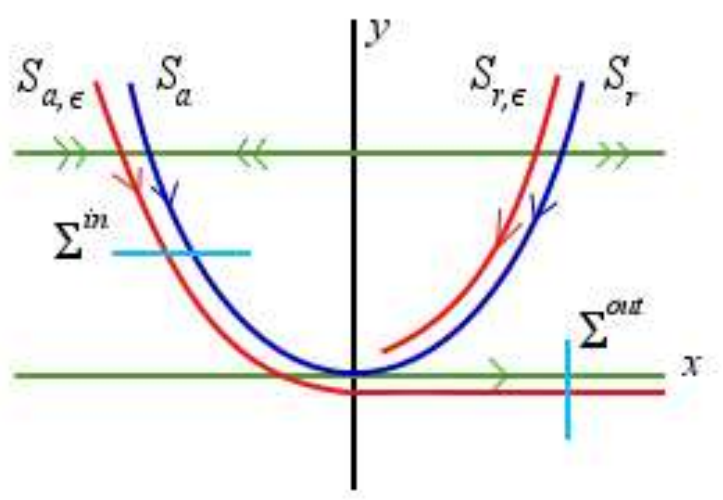

Figure A.2. Slow manifolds and sections.

dynamics close to the fold point is governed by equations of the form

$$
\begin{aligned}
& x^{\prime}=x^{2}-y+O\left(\varepsilon, x y, y^{2}, x^{3}\right), \\
& y^{\prime}=\varepsilon(-1+O(x, y, \varepsilon)), \\
& \varepsilon^{\prime}=0 .
\end{aligned}
$$

The degenerate equilibrium $(0,0,0)$ of system (A.4) is blown-up to a sphere by the transformation

$$
x=r \bar{x}, \quad y=r^{2} \bar{y}, \quad \varepsilon=r^{3} \bar{\varepsilon}
$$

with $(\bar{x}, \bar{y}, \bar{\varepsilon}) \in \mathbb{S}^{2}$ and $r \in \mathbb{R}$. After dividing out a suitable power of the radial variable $r$, a nontrivial flow on the sphere is obtained. The resulting flow on the sphere is sufficiently non-degenerate to allow a complete analysis. For details we refer to $[13]$.

In order to describe the behavior of the attracting slow manifold $S_{a, \varepsilon}$ beyond the fold point we consider a section $\Sigma^{i n}$ transverse to $S_{a}$ defined by $y=y_{0}, y_{0}>0$ and a section $\Sigma^{\text {out }}$ transverse to the unstable fiber of the fold point defined by $x=x_{0}, x_{0}>0$. The following result has been proven as Theorem 2.1 in [13].

THEOREM A.1. Under the assumptions made in this section there exists $\varepsilon_{0}>0$ such that the following assertions hold for $\varepsilon \in\left(0, \varepsilon_{0}\right]$ :

1. The manifold $S_{a, \varepsilon}$ passes through $\Sigma^{\text {out }}$ at a point $\left(x_{0}, h(\varepsilon)\right)$ where $h(\varepsilon)=$ $O\left(\varepsilon^{2 / 3}\right)$.

2. Under the flow of system (A.1) the section $\Sigma^{i n}$ is mapped to an interval around $S_{a, \varepsilon} \cap \Sigma^{\text {out }}$. The transition map from $\Sigma^{i n}$ to $\Sigma^{\text {out }}$ is a contraction with contraction rate $e^{-c / \varepsilon}$, where $c$ is a positive constant.

We conclude that for $\varepsilon$ small solutions starting between $S_{a, \varepsilon}$ and $S_{r, \varepsilon}$ are exponentially contracted onto $S_{a, \varepsilon}$, follow the slow flow on $S_{a, \varepsilon}$ downwards and jump almost horizontally to the right after passing the fold point.

REMARK A.1. In the situation of relaxation oscillations described in Section 2.2 Theorem A.1 readily implies the existence of relaxation cycles for $\varepsilon$ small obtained as fixed points of a Poincaré map which is defined as the composition of two maps of the type described in the theorem, see ([14]) for details.

\section{REFERENCES}


[1] F. Dumortier and R. Roussarie, Canard cycles and center manifolds, Mem. Amer. Math. Soc., Providence, 577 (1996).

[2] F. Dumortier and R. Roussarie, Multiple canard cycles in generalized Liénard equations, J. Differential Equations, 174 (2001), pp. 1-29.

[3] F. Dumortier, N. Popovic, And T. J. Kaper, The critical wave speed for the FisherKolmogorov-Petrowskii-Piscounov equation with cut-off, Nonlinearity, 20 (2007), pp. 855877.

[4] N. Fenichel, Geometric singular perturbation theory, J. Differential Equations, 31 (1979), pp. $53-98$.

[5] S. Van Gils, M. Krupa, and P. Szmolyan, Asymptotic expansions using blow-up, Z. Angew. Math. Phys., 56 (2005), pp. 369-397.

[6] J. Grasman, Asymptotic Methods for Relaxation Oscillations and Applications, Springer, New York, 1987.

[7] J. Guckenheimer and P. Holmes, Nonlinear Oscillations, Dynamical Systems, and Bifurcations of Vector Fields, Springer, New York, 1983.

[8] I. Gucwa ANd P. Szmolyan Geometric singular perturbation analysis of an Autocatalotor model, to appear in Discrete and Continuous Dynamical Systems - Series S, 2009.

[9] A. Huber and P. Szmolyan, Geometric singular perturbation analysis of the Yamada model, SIAM J. Appl. Dyn. Syst., 4 (2005), pp. 607-648.

[10] C.K.R.T. Jones, Geometric singular perturbation theory, Springer Lecture Notes in Mathematics, Berlin, 1609 (1995), pp. 44-120.

[11] J. Kevorkian and J. D. Cole, Multiple scale and singular perturbation methods, Applied Mathematical Sciences, 114. Springer-Verlag, New York, 1996.

[12] M. Krupa, N. Popovic, And N. Kopeld, Mixed-mode oscillations in three time-scale systems: a prototypical example, SIAM J. Appl. Dyn. Syst., 7 (2008), pp. 361-420.

[13] M. KRupa AND P. Szmolyan, Extending geometric singular perturbation theory to nonhyperbolic points-fold and canard points in two dimensions, SIAM J. Math. Analysis, 33 (2001), pp. 286-314.

[14] M. Krupa And P. Szmolyan, Relaxation oscillation and canard explosion, J. Differential Equations, 174 (2001), pp. 312-368.

[15] P. De Maesschalck and F. Dumortier, Canard solutions at non-generic turning points, Trans. Amer. Math. Soc., 358 (2006), pp. 2291-2334.

[16] E.F. Mishchenko And N.Kh. Rozov, Differential Equations with Small Parameters and Relaxation Oscillations, Plenum Press, New York, 1980.

[17] R. E. O'Malley, Introduction to singular perturbations, Academic Press, New York, 1974.

[18] N. Popovic And P. Szmolyan, A geometric analysis of the Lagerstrom model problem, J. Differential Equations, 199 (2004), pp. 290-325.

[19] S. Schecter and P. Szmolyan, Persistence of rarefactions under Dafermos regularization: blow-up and an exchange lemma for gain-of-stability turning points, SIAM J. Appl. Dyn. Syst., to appear.

[20] L. Segel, A. Goldbeter, Scaling in biochemical kinetics: dissection of a relaxation oscillator, J. Math. Biol., 32 (1994), pp. 147-160.

[21] P. Szmolyan and M. Wechselberger, Canards in $\mathbb{R}^{3}$, J. Differential Equations, 177 (2001), pp. 419-453.

[22] P. Szmolyan and M. Wechselberger, Relaxation oscillations in $\mathbb{R}^{3}$, J. Differential Equations, 200 (2004), pp. 69-104. 